\title{
Robust finite volume schemes for two-fluid plasma equations
}

\author{
Abgrall, Rémi ; Kumar, Harish
}

\begin{abstract}
Two-fluid plasma equations are derived by taking moments of Boltzmann equations. Ignoring collisions and viscous terms and assuming local thermodynamic equilibrium we get five moment equations for each species (electrons and ions), known as two-fluid plasma equations. These equations allow different temperatures and velocities for electrons and ions, unlike ideal magnetohydrodynamics equations. In this article, we present robust second order MUSCL schemes for two-fluid plasma equations based on Strang splitting of the flux and source terms. The source is treated both explicitly and implicitly. These schemes are shown to preserve positivity of the pressure and density. In the case of explicit treatment of source term, we derive explicit condition on the time step for it to be positivity preserving. The implicit treatment of the source term is shown to preserve positivity, unconditionally. Numerical experiments are presented to demonstrate the robustness and efficiency of these schemes.
\end{abstract}

DOI: https://doi.org/10.1007/s10915-013-9809-6

Posted at the Zurich Open Repository and Archive, University of Zurich

ZORA URL: https://doi.org/10.5167/uzh-147700

Journal Article

Accepted Version

Originally published at:

Abgrall, Rémi; Kumar, Harish (2014). Robust finite volume schemes for two-fluid plasma equations. Journal of Scientific Computing, 60(3):584-611.

DOI: https://doi.org/10.1007/s10915-013-9809-6 


\title{
ROBUST FINITE VOLUME SCHEMES FOR TWO-FLUID PLASMA EQUATIONS
}

\author{
REMI ABGRALL AND HARISH KUMAR
}

\begin{abstract}
Two-fluid plasma equations are derived by taking moments of Boltzmann equations. Ignoring collisions and viscous terms and assuming local thermodynamic equilibrium we get five moment equations for each species (electrons and ions), known as two-fluid plasma equations. These equations allow different temperatures and velocities for electrons and ions, unlike ideal magnetohydrodynamics (MHD) equations.

In this article, we present robust second order MUSCL schemes for two-fluid plasma equations based on strang-splitting of the flux and source terms. The source is treated both explicitly and implicitly. These schemes are shown to preserve positivity of the pressure and density. In the case of explicit treatment of source term, we derive explicit condition on the time step for it to be positivity preserving. The implicit treatment of the source term is shown to preserve positivity, unconditionally. Numerical experiments are presented to demonstrate the robustness and efficiency of these schemes.
\end{abstract}

\section{INTRODUCTION}

Equations of magnetohydrodynamics (MHD) are frequently used to model plasma flows. These equations combine the Euler equations for compressible flow with Maxwell's equations for magnetic fields and assume quasi-neutrality of the plasma i.e. the difference in number density of ions and electrons is ignored. However, in many applications like fast magnetic reconnection, the assumption of quasi-neutrality is violated. In such cases, one resorts to extended MHD models. A popular model consider the flow of two different species, one electron and one ion, separately. 
This results in the so-called two fluid plasma equations (see [9], [15]). In non-dimensional conservative variables these equations can be written as:

$$
\begin{aligned}
\frac{\partial \rho_{i}}{\partial t}+\nabla \cdot\left(\rho_{i} \mathbf{v}_{i}\right) & =0 \\
\frac{\partial\left(\rho_{i} \mathbf{v}_{i}\right)}{\partial t}+\nabla \cdot\left(\rho_{i} \mathbf{v}_{i} \mathbf{v}_{i}^{\top}+p_{i} \mathbf{I}\right) & =\frac{1}{\hat{l}_{r}} \rho_{i}\left(\mathbf{E}+\mathbf{v}_{i} \times \mathbf{B}\right), \\
\frac{\partial \mathcal{E}_{i}}{\partial t}+\nabla \cdot\left(\left(\mathcal{E}_{i}+p_{i}\right) \mathbf{v}_{i}\right) & =\frac{1}{\hat{l}_{r}} \rho_{i}\left(\mathbf{E} \cdot \mathbf{v}_{i}\right), \\
\frac{\partial \rho_{e}}{\partial t}+\nabla \cdot\left(\rho_{e} \mathbf{v}_{e}\right) & =0, \\
\frac{\partial\left(\rho_{e} \mathbf{v}_{e}\right)}{\partial t}+\nabla \cdot\left(\rho_{e} \mathbf{v}_{e} \mathbf{v}_{e}^{\top}+p_{e} \mathbf{I}\right) & =-\frac{m}{\hat{l}_{r}} \rho_{e}\left(\mathbf{E}+\mathbf{v}_{e} \times \mathbf{B}\right), \\
\frac{\partial \mathcal{E}_{e}}{\partial t}+\nabla \cdot\left(\left(\mathcal{E}_{e}+p_{e}\right) \mathbf{v}_{e}\right) & =-\frac{m}{\hat{l}_{r}} \rho_{e}\left(\mathbf{E} \cdot \mathbf{v}_{e}\right), \\
\frac{\partial \mathbf{B}}{\partial t}+\nabla \times \mathbf{E}+\kappa \nabla \psi & =0, \\
\frac{\partial \mathbf{E}}{\partial t}-\hat{c}^{2} \nabla \times \mathbf{B}+\xi \hat{c}^{2} \nabla \phi & =-\frac{1}{\hat{\lambda}_{d}^{2} \hat{l}_{r}}\left(r_{i} \rho_{i} \mathbf{v}_{i}+r_{e} \rho_{e} \mathbf{v}_{e}\right), \\
\frac{\partial \phi}{\partial t}+\xi \nabla \cdot \mathbf{E} & =\frac{\xi}{\hat{\lambda}_{d}^{2} \hat{l}_{r}}\left(r_{i} \rho_{i}+r_{e} \rho_{e}\right), \\
\frac{\partial \psi}{\partial t}+\kappa \hat{c}^{2} \nabla \cdot \mathbf{B} & =0 .
\end{aligned}
$$

Here, the subscript $\{i, e\}$ refers to the ion and electron species respectively, $\rho_{\{i, e\}}$ are densities, $\mathbf{v}_{\{i, e\}}=\left(v_{\{i, e\}}^{x}, v_{\{i, e\}}^{y}, v_{\{i, e\}}^{z}\right)$ are velocities, $\mathcal{E}_{\{i, e\}}$ are the energies, $p_{\{i, e\}}$ are the pressures, $\mathbf{B}=$ $\left(B^{x}, B^{y}, B^{z}\right)$ is the magnetic field, $\mathbf{E}=\left(E^{x}, E^{y}, E^{z}\right)$ is the electric field, $\phi, \psi$ are the potentials and $\xi, \kappa$ are the speeds for Maxwell equations. Also, $r_{\alpha}=q_{\alpha} / m_{\alpha}, \quad \alpha \in\{i, e\}$ are the charge-mass ratios and $m=m_{i} / m_{e}$ is the ion-electron mass ratio.

Several physically significant parameters appear in the non-dimensionalized form (1.1). Here, $\hat{l}_{r}=\frac{l_{r}}{x_{0}}=\frac{m_{i} v_{i}^{T}}{q_{i} B_{0} x_{0}}$ is the normalized ion Larmor radius, $\hat{c}=c / v_{i}^{T}$ is the normalized speed of light and $\hat{\lambda}_{d}=\lambda_{d} / l_{r}=\sqrt{\epsilon_{0} v_{i}^{T 2} / n_{0} q_{i}} / l_{r}$ is the ion Debye length normalized with Larmor radius. Also, $v_{i}^{T}$ is the reference thermal velocity of ions, $B_{0}$ is the reference magnetic field and $x_{0}$ is the reference length. Ion mass $m_{i}$ is assumed to be 1 . In addition, we assume that both the ion and the electron satisfies following ideal gas law:

$$
\mathcal{E}_{\alpha}=\epsilon_{\alpha}+\frac{1}{2} \rho_{\alpha}\left|\mathbf{v}_{\alpha}\right|^{2}, \quad \epsilon_{\alpha}=\frac{p_{\alpha}}{\gamma-1}, \quad \alpha \in\{i, e\} .
$$

with $\gamma=5 / 3$. Eqns. (1.1a)-(1.1c) represent the conservation of mass, momentum and energy for the ions. The source term in (1.1b) is Lorentz force acting on ions due to the electric and magnetic fields. Similarly, Eqns.(1.1d)-(1.1f) represent conservation of mass momentum and energy for electrons. Eqns. (1.1g)-(1.1j) are the perfectly hyperbolic Maxwell's (PHM) equations (see [14]). These equations satisfy the divergence constraint approximately and are consistent with the hyperbolic structure of the fluid equations. Note that, when the Larmor radius $\hat{l}_{r} \rightarrow 0$, the two-fluid model approaches the MHD limit. Similarly, for $\hat{l}_{r} \rightarrow \infty$, (1.1) reduces to Euler equations for ions and electrons.

The system of two-fluid plasma Eqns. (1.1) is a system of balance laws of the form,

$$
\mathbf{u}_{t}+\nabla \cdot \mathbf{f}(\mathbf{u})=\mathbf{s}(\mathbf{u})
$$


with, the vector of non-dimensional conservative variables,

$$
\mathbf{u}=\left\{\rho_{i}, \rho_{i} \mathbf{v}_{i}, \mathcal{E}_{i} \rho_{e}, \rho_{e} \mathbf{v}_{e}, \mathcal{E}_{e} \mathbf{B}, \mathbf{E}, \phi, \psi\right\}^{\top} .
$$

The flux can be divided into three independent parts as,

$$
\mathbf{f}(\mathbf{u})=\left\{\begin{array}{c}
\mathbf{f}_{i}\left(\mathbf{u}_{i}\right) \\
\mathbf{f}_{e}\left(\mathbf{u}_{e}\right) \\
\mathbf{f}_{m}\left(\mathbf{u}_{m}\right)
\end{array}\right\} \quad \text { with } \quad \mathbf{f}_{\alpha}=\left\{\begin{array}{c}
\rho_{\alpha} \mathbf{v}_{\alpha} \\
\rho_{\alpha} \mathbf{v}_{\alpha} \mathbf{v}_{\alpha}^{\top}+p_{\alpha} \mathbf{I} \\
\left(\mathcal{E}_{\alpha}+p_{\alpha}\right) \mathbf{v}_{\alpha}
\end{array}\right\}
$$

where $\mathbf{u}_{\alpha}=\left\{\rho_{\alpha}, \rho_{\alpha} \mathbf{v}_{\alpha}, \mathcal{E}_{\alpha}\right\}^{\top}, \alpha \in\{i, e\}, \mathbf{u}_{m}=\{\mathbf{B}, \mathbf{E}, \phi, \psi\}$ and $\mathbf{I}$ is a $3 \times 3$ identity matrix. Maxwell flux is given by,

$$
\mathbf{f}_{m}\left(\mathbf{u}_{m}\right)=\left\{\begin{array}{c}
\mathcal{T}(\mathbf{E})+\kappa \psi \mathbf{I} \\
-\hat{c}^{2} \mathcal{T}(\mathbf{B})+\xi \hat{c}^{2} \phi \mathbf{I} \\
\xi \mathbf{E} \\
\kappa \hat{c}^{2} \mathbf{B}
\end{array}\right\} \text {, where } \mathcal{T}(\mathbf{A})=\left[\begin{array}{ccc}
0 & A^{z} & -A^{y} \\
-A^{z} & 0 & A^{x} \\
A^{y} & -A^{x} & 0
\end{array}\right],
$$

for any vector $\mathbf{A}=\left(A^{x}, A^{y}, A^{z}\right)$. These three components are coupled via source term,

$$
\mathbf{s}(\mathbf{u})=\left\{\begin{array}{c}
0 \\
\frac{1}{\hat{l}_{r}} \rho_{i}\left(\mathbf{E}+\mathbf{v}_{i} \times \mathbf{B}\right) \\
\frac{1}{\hat{l}_{r}} \rho_{i}\left(\mathbf{E} \cdot \mathbf{v}_{i}\right) \\
0 \\
-\frac{m}{\hat{l}_{r}} \rho_{e}\left(\mathbf{E}+\mathbf{v}_{e} \times \mathbf{B}\right) \\
-\frac{m}{\hat{l}_{r}} \rho_{e}\left(\mathbf{E} \cdot \mathbf{v}_{e}\right) \\
\mathbf{0}_{3 \times 1} \\
-\frac{1}{\hat{\lambda}_{d}^{2} \hat{l}_{r}}\left(r_{i} \rho_{i} \mathbf{v}_{i}+r_{e} \rho_{e} \mathbf{v}_{e}\right) \\
\frac{\xi}{\hat{\lambda}_{d}^{2} \hat{l}_{r}}\left(r_{i} \rho_{i}+r_{e} \rho_{e}\right) \\
0 \\
0
\end{array}\right\}
$$

We are interested in the solutions $\mathbf{u}: \mathbb{R}^{3} \times \mathbb{R}^{+} \rightarrow \Omega$, of (1.3), belonging to the physically admissible set,

$$
\Omega=\left\{\mathbf{u} \in \mathbb{R}^{18} ; \quad \rho_{\alpha}>0 \text { and } \epsilon_{\alpha}>0 \text { for } \alpha \in\{i, e\}\right\} .
$$

First, we state the following result,

Lemma 1.1. The system (1.3) is hyperbolic for all $\mathbf{u} \in \Omega$ with the eigenvalues,

$$
\mathbf{v}_{\alpha} \pm a_{\alpha}, \quad \mathbf{v}_{\alpha}, \quad \alpha \in\{i, e\}, \pm c, \pm \xi c, \quad \pm \kappa c .
$$

with $a_{\alpha}=\sqrt{\gamma p_{\alpha} / \rho_{\alpha}}$. Furthermore, the eigenvalues $\mathbf{v}_{\alpha} \pm a_{\alpha}$ is associated with a genuinely nonlinear fields, while all other eigenvalues are associated with linearly degenerate fields.

Proof. The proof follows from analysis of Euler equations and perfectly hyperbolic Maxwell equations.

Note that, if we ignore source terms, than the physical solutions of (1.3) are in $\Omega$. Next result shows that, this is also true when we take source terms in to considerations.

Lemma 1.2. The source term is $\Omega$-invariant i.e. given initial condition $\mathbf{u}(0) \in \Omega$, the solution $\mathbf{u}(t)$ of source $O D E$

$$
\frac{d \mathbf{u}}{d t}=\mathbf{s}(\mathbf{u})
$$

is in $\Omega$. Furthermore, the source terms are oscillatory and energy conservative. 
Proof. Let us ignore the convection part and consider the ODE,

$$
\frac{d \mathbf{u}}{d t}=\mathbf{s}(\mathbf{u})
$$

A straight forward calculation shows that,

$$
\frac{d \rho_{\alpha}}{d t}=0 \quad \text { and } \quad \frac{d \epsilon_{\alpha}}{d t}=0, \quad \alpha \in\{i, e\} .
$$

which implies source terms are $\Omega$-invariants. Also, not all the component of $\mathbf{u}$ have source terms. Considering only the components which have source terms by defining, $\mathbf{u}_{1}=\left\{\mathbf{v}_{i}, \mathbf{v}_{e}, \mathbf{E}\right\}$,

$$
\frac{d \mathbf{u}_{1}}{d t}=\mathbf{s}_{1}\left(\mathbf{u}_{1}\right), \text { with } \quad \mathbf{s}_{1}\left(\mathbf{u}_{1}\right)=\left(\begin{array}{c}
\frac{1}{\hat{l}_{r}}\left(\mathbf{E}+\mathbf{v}_{i} \times \mathbf{B}\right) \\
-\frac{m}{\hat{l}_{r}}\left(\mathbf{E}+\mathbf{v}_{e} \times \mathbf{B}\right) \\
-\frac{1}{\hat{\lambda}_{d}^{2} \hat{l}_{r}}\left(r_{i} \rho_{i} \mathbf{v}_{i}+r_{e} \rho_{e} \mathbf{v}_{e}\right)
\end{array}\right) .
$$

Also, if

$$
\bar{E}=\frac{\rho_{i} \mathbf{v}_{i}^{2}+\rho_{e} \mathbf{v}_{e}^{2}+\hat{\lambda}_{d}^{2} \mathbf{E}^{2}}{2}
$$

then a simple calculation shows that,

$$
\frac{d \bar{E}}{d t}=0 .
$$

Furthermore, one eigenvalue of jacobian $\partial_{\mathbf{u}_{1}} \mathbf{s}_{1}\left(\mathbf{u}_{1}\right)$ is zero and all other eigenvalues are purely imaginary (see $[9,21]$ ).

As system is hyperbolic, solutions may develop discontinuities in finite time. To, choose physically meaningful solution, we supplement (1.3) with additional admissible criteria known as the entropy condition. Following [12], we introduce fluid entropies,

$$
e_{\alpha}=-\frac{\rho_{\alpha} s_{\alpha}}{\gamma-1} \text { with } s_{\alpha}=\log p_{\alpha}-\gamma \log \rho_{\alpha}, \quad \alpha \in\{i, e\} .
$$

Now we have following entropy stability:

Lemma 1.3. The smooth solutions of (1.3) satisfies,

$$
\partial_{t} e_{\alpha}+\nabla \cdot q_{\alpha}=0, \quad q_{\alpha}=\mathbf{v}_{\alpha} e_{\alpha}
$$

Furthermore, the weak solutions of (1.3) will satisfies the entropy inequality,

$$
\partial_{t} e_{\alpha}+\nabla \cdot q_{\alpha} \leq 0 \text {. }
$$

Proof. The proof follows from the observation that the source terms do not effect pressure evolution. For smooth solutions, we have,

$$
\partial_{t} p_{\alpha}+\gamma p_{\alpha} \nabla \cdot \mathbf{v}_{\alpha}+\mathbf{v}_{\alpha} \cdot \nabla p_{\alpha}=0 .
$$

Combining this with density advection we arrives at (1.12) for smooth solutions. For non-smooth solutions (1.12) implies (1.13). See [12] for more details.

Remark 1.4. We note that (1.13) can be used to derive energy estimates for (1.3). We refer to [12] for further details. 
Numerical methods to solve (1.3) are based on the finite volume methods [11]. These methods are based on the evolving the cell averages of the conservative variables in time by approximating the flux through the cell interfaces. The higher order accuracy in space can be obtained by reconstructing the solution using interpolation procedure based on TVD limiters, ENO and WENO. Strong stability preserving (SSP) schemes are used for higher order accuracy in time. Although, two fluid equations are a system of balance laws, they present significant difficulties for these methods. These are:

- The system has stiffness in both flux and source term. In the flux, the stiffnesses are due to high normalized speed of light $\hat{c}$ and electron sound speed $a_{e}$. The source is stiff in the case of low normalized Larmor radius (i.e. high charge to mass ratio).

- The source terms are oscillatory. So, to obtain a robust numerical scheme we need a carefully treatment of source.

- We have shown that physically meaningful solution are $\Omega$-invariant. In addition, entropy stability holds with source terms. However, there is no guarantee that a general numerical scheme will have these properties.

Due to these challenges, few numerical method exists for two-fluid equations with provable stability. In [15], the authors derive a Roe-type Riemann solver. Time updates are performed by treating the stiff source term implicitly and the flux terms explicitly. The resulting non-linear equations are solved using Newton iterations. This method might be diffusive and may require many iterations for each time step. In [9], the authors propose a wave propagation method (see [11]) for the spatial discretization. For time updates, a second-order operator splitting approach is used. A similar approach is taken in [13, 10], where spatial discretization is based on discontinuous Galerkin (DG) methods and time update is based on SSP-RK methods. Both of these approaches are easy to implement but can be computationally expensive, especially for realistic charge to mass ratios. More recently, in [12] authors have presented entropy stable schemes for the twofluid equations. The source terms are treated implicitly and resulting algebraic equations are solved exactly. However, schemes still do not guarantee the positivity of density and internal energy.

In this article, we propose numerical schemes with following properties:

- The schemes proposed here are based on the splitting the Equation (1.3) in to flux and source contributions. The homogeneous part contains only flux contribution and can be discretize using the numerical scheme presented in $[1,2,3]$. In particular, we follow [3] which ensures that the updated homogeneous solutions still preserve the positivity of density and pressure. In Section 2.1, we present a brief overview of these schemes in the context of two-fluid equations.

- Note that the source terms are stiff and oscillatory. So, a simple source discretization may not preserve the positivity of density and pressure. The key contribution of this article is to analyze explicit and implicit source discretizations and derive the conditions under which they preserve positivity of density and pressure. This is presented in Section 2.2. In the explicit scheme case, we derive condition on time step for scheme to preserve positivity of density and pressure (See Theorem 2.3). For implicit scheme, we have shown that the source update preserve positivity of density and pressure unconditionally (see Theorem 2.4). Furthermore, the implicit treatment of the source update also overcome the stiffness due to low normalized Larmor radius.

- Combining both flux and source update via strang splitting, we show that in general any scheme for Euler equations which preserve the positivity of density and pressure can be extended to two-fluid equations using the source discretizations proposed here. 
The rest of the article is organized as follows: In the following Section 2, we present finite volume schemes which preserve the positivity of density and pressure. The first part (See Section 2.1) consists of numerical schemes for homogeneous part which are simple extension of numerical schemes of [3] for Euler equations to two-fluid equations. The critical second part (See Section 2.2) of this Section contains discretizations of the source term and related analysis. Section 3 is dedicated to the numerical experiments, where we show the robustness and efficiency of the schemes proposed in Section 2.

\section{Numerical Schemes}

In this section, we will present schemes for two-fluid equations which preserve the positivity of density and pressure. For simplicity, we will consider the Equations (1.3) in one dimension. We still have a complete system with eighteen equations, unlike ideal MHD equations (where we have loose equations for $B^{x}$ due to divergence free condition of magnetic field). Furthermore, source does not change when we consider one dimensional version of the Equations (1.3). So, the extension to two and three dimensions is straight forward. 1

In finite volume methods, a piecewise constant approximation of the solution $\mathbf{u}\left(x, t^{n}\right)$ at time $t^{n}$ is based on the cell averages,

$$
\mathbf{w}_{i}^{n}=\frac{1}{\Delta x} \int_{x_{i-\frac{1}{2}}}^{x_{i+\frac{1}{2}}} \mathbf{u}\left(x, t^{n}\right) d x, \quad x \in\left(x_{i-\frac{1}{2}}, x_{i+\frac{1}{2}}\right),
$$

where $\left(x_{i}\right)_{i \in \mathbb{Z}}$ are the cell centers of the cells $I_{i}=\left(x_{i-\frac{1}{2}}, x_{i+\frac{1}{2}}\right)$ and $x_{i+\frac{1}{2}}=x_{i}+\left(x_{i+1}-x_{i}\right) / 2$. For the simplicity, we will consider uniform mesh, i.e. $\Delta x=x_{i+1}-x_{i}$ is same for all $i \in \mathbb{Z}$. As we are interested in physically meaningful solutions only, let us assume that given solution $\mathbf{w}_{i}^{n} \in \Omega$ for all $i \in \mathbb{Z}$. We now present schemes which ensures that $\mathbf{w}_{i}^{n+1} \in \Omega$. We need following definition:

Definition 2.1 ( $\Omega$-Invariance). An update $\mathcal{M}$ of the given solution $\mathbf{w}_{i}^{n} \in \Omega$, for all $i \in \mathbb{Z}$, is said to $\Omega$-invariant, if updated solution $\mathbf{w}_{i}^{n+1}=\mathcal{M}\left(\mathbf{w}_{i}^{n}\right)$ belongs to $\Omega$.

In order to approximating the solutions of (1.3), we will split flux and source. Let's first consider the discretization without the source term.

2.1. Discretization of the Homogeneous problem. The one dimensional homogeneous version of $(1.3)$ is,

$$
\partial_{t} \mathbf{u}+\partial_{x} \mathbf{f}^{x}(\mathbf{u})=0
$$

where $\mathbf{f}^{x}$ is $\mathbf{x}$-component of the flux $\mathbf{f}$. A first order finite volume conservative scheme for the $(2.2)$ is of the form,

$$
\mathbf{w}_{i}^{n+1}=\mathbf{w}_{i}^{n}-\frac{\Delta t}{\Delta x}\left(\mathbf{F}^{x}\left(\mathbf{w}_{i}^{n}, \mathbf{w}_{i+1}^{n}\right)-\mathbf{F}^{x}\left(\mathbf{w}_{i-1}^{n}, \mathbf{w}_{i}^{n}\right)\right),
$$

where $\mathbf{F}^{x}: \Omega \times \Omega \rightarrow \mathbb{R}^{18}$ is a Lipschitz continuous, conservative numerical flux function, consistent with $\mathbf{f}^{x}$. We will denote update (2.3) as,

$$
\mathbf{w}_{i}^{n+1}=\mathcal{H}_{\Delta t}^{1} \mathbf{w}_{i}^{n} .
$$

We will now describe the flux function $\mathbf{F}^{x}$. 
2.1.1. HLLC based numerical flux for two-fluid equations. As the flux $\mathbf{f}^{x}$ has three independent parts, a numerical flux for two-fluid equations will have the form,

$$
\mathbf{F}^{x}\left(\mathbf{w}_{l}, \mathbf{w}_{r}\right)=\left(\begin{array}{c}
\mathbf{F}_{i}^{x}\left(\mathbf{w}_{i, l}, \mathbf{w}_{i, r}\right) \\
\mathbf{F}_{e}^{x}\left(\mathbf{w}_{e, l}, \mathbf{w}_{e, r}\right) \\
\mathbf{F}_{m}^{x}\left(\mathbf{w}_{m, l}, \mathbf{w}_{m, r}\right)
\end{array}\right) .
$$

Here, $\mathbf{F}_{\alpha}^{x}, \alpha \in\{i, e\}$ are the numerical flux functions for Euler equations and $\mathbf{F}_{m}$ is the numerical flux for the Maxwell's equations. In general, we can combine any numerical fluxes of Euler equations and Maxwell's equations to obtain a numerical flux function two-fluid equations. Note that the any choice of numerical flux function $\mathbf{F}_{m}^{x}$ of the Maxwell's equations will have no effect on the positivity and entropy stability of the scheme (2.3) for Equations (2.2). Furthermore, note that the Maxwell's equations are linear. So, the Maxwell component of (2.2) can be written as,

$$
\partial_{t} \mathbf{u}_{m}+\partial_{x} \mathbf{f}_{m}^{x}\left(\mathbf{u}_{m}\right)=0 \quad \text { with } \quad \mathbf{f}_{m}^{x}\left(\mathbf{u}_{m}\right)=\mathbf{A}_{m}^{x} \mathbf{u}_{m}
$$

We will use HLLE solver for the numerical flux corresponding to $\mathbf{f}_{m}^{x}$.

For the fluid part, we will consider the HLLC solver for the Euler equations. Let us consider the Euler equation in one dimensions with all the components of velocity,

$$
\partial_{t} \tilde{\mathbf{u}}+\partial_{x} \mathbf{g}(\tilde{\mathbf{u}})=0, \quad \mathbf{g}(\tilde{\mathbf{u}})=\left(\begin{array}{c}
\rho v^{x} \\
\rho v^{x}+p \\
\rho v^{x} v^{y} \\
\rho v^{x} v^{z} \\
(\mathcal{E}+p) v^{x}
\end{array}\right)
$$

with $\tilde{\mathbf{u}}=\left\{\rho, \rho v^{x}, \rho v^{y}, \rho v^{z}, \mathcal{E}\right\}$ with equation of state given by (1.2). The HLLC numerical flux for the Euler equation is based on the approximate Riemann solver containing two intermediate states, i.e. given sates $\tilde{\mathbf{w}}_{l}$ and $\tilde{\mathbf{w}}_{r}$, we consider the solution of the form,

$$
\tilde{\mathbf{w}}(x, t)= \begin{cases}\tilde{\mathbf{w}}_{l}, & \text { if } \quad b_{l}>0, \\ \tilde{\mathbf{w}}_{l}^{*}, & \text { if } \quad b_{l} \leq 0 \leq b^{*}, \\ \tilde{\mathbf{w}}_{r}^{*}, & \text { if } \quad b^{*} \leq 0 \leq b_{r}, \\ \tilde{\mathbf{w}}_{r} & \text { if } \quad b_{r}<0 .\end{cases}
$$

To calculate states $\tilde{\mathbf{w}}_{l, r}^{*}$, we will use Rankine-Hugoniot conditions across the discontinuities. Clearly, there are more unknowns than the number of equations. To overcome this, we introduce additional conditions, which are satisfied by the exact solution.

First note that for the tangential velocity components we have,

$$
\begin{array}{cc}
v_{l}^{y *}=v_{l}^{y}, & v_{r}^{y *}=v_{r}^{y}, \\
v_{l}^{z *}=v_{l}^{z}, & v_{r}^{z *}=v_{r}^{z},
\end{array}
$$

Also, as $\tilde{\mathbf{w}}_{l}^{*}$ and $\tilde{\mathbf{w}}_{r}^{*}$ are separated by contact discontinuity, so,

$$
\begin{array}{r}
p_{l}^{*}=p_{r}^{*}=p^{*}, \\
v_{l}^{x *}=v_{r}^{x *}=v^{x *} .
\end{array}
$$

It is reasonable to consider the speed of contact discontinuity to be $b^{*}=v^{x *}$ (see [19]). Using these relations, we will now derive expressions for states $\tilde{\mathbf{w}}_{l}^{*}$ and $\tilde{\mathbf{w}}_{r}^{*}$. Note that,

$$
p_{l}^{*}=p_{l}+\rho_{l}\left(b_{l}-v_{l}^{x}\right)\left(v^{x *}-v_{l}^{x}\right) \text { and } p_{r}^{*}=p_{r}+\rho_{r}\left(b_{r}-v_{r}^{x}\right)\left(v^{x *}-v_{r}^{x}\right) .
$$

Using pressure equality (2.10) over contact discontinuity,

$$
v^{x *}=\frac{p_{r}-p_{l}+\rho_{l} v_{l}^{x}\left(b_{l}-v_{l}^{x}\right)-\rho_{r} v_{r}^{x}\left(b_{r}-v_{r}^{x}\right)}{\rho_{l}\left(b_{l}-v_{l}^{x}\right)-\rho_{r}\left(b_{r}-v_{r}^{x}\right)} .
$$


Expression for $p^{*}$ can be found using (2.11). Now we need to obtain expression for $\rho_{l}^{*}$ and $\rho_{r}^{*}$, which can be done by using Rankine-Hugoniot condition across the wave $b_{l}$ and $b_{l}$. Finally the numerical flux $\mathbf{G}^{x}$ is given by,

$$
\mathbf{G}_{h l l c}^{x}=\left\{\begin{array}{l}
\mathbf{g}\left(\tilde{\mathbf{w}}_{l}\right), \quad \text { if } \quad b_{l}>0, \\
\mathbf{g}\left(\tilde{\mathbf{w}}_{l}^{*}\right), \quad \text { if } \quad b_{l} \leq 0 \leq v^{x *}, \\
\mathbf{g}\left(\tilde{\mathbf{w}}_{r}^{*}\right), \quad \text { if } \quad v^{x *} \leq \leq \leq b_{r}, \\
\mathbf{g}\left(\tilde{\mathbf{w}}_{r}\right), \quad \text { if } \quad b_{r}<0 .
\end{array}\right.
$$

This flux is shown to have positivity preserving property (see [20]) if we take,

$$
b_{l} \leq v_{l}^{x}-a_{l}, \quad \text { and } \quad b_{r} \geq v_{r}^{x}+a_{r} .
$$

We choose,

$$
b_{l}=\min \left(v_{l}^{x}-a_{l}, v_{m}^{x}-a_{m}\right) \quad b_{r}=\max \left(v_{r}^{x}+a_{r}, v_{m}^{x}+a_{m}\right) .
$$

Here, state $\tilde{\mathbf{w}}_{m}$ is based on simple average on primitive variables of states $\tilde{\mathbf{w}}_{l}$ and $\tilde{\mathbf{w}}_{r}$. One can also consider Roe averages. Combining all the components, the numerical flux for the homogeneous Equations (2.2) is given by,

$$
\mathbf{F}^{x}\left(\mathbf{w}_{l}, \mathbf{w}_{r}\right)=\left(\begin{array}{c}
\mathbf{G}_{h l l c}^{x}\left(\mathbf{w}_{i, l}, \mathbf{w}_{i, r}\right) \\
\mathbf{G}_{h l l c}^{x}\left(\mathbf{w}_{e, l}, \mathbf{w}_{e, r}\right) \\
\mathbf{F}_{m, h l l e}^{x}\left(\mathbf{w}_{m, l}, \mathbf{w}_{m, r}\right)
\end{array}\right) .
$$

In addition to the HLLC flux for the flow part, we will also consider HLLE and Rusanov numerical fluxes. These fluxes also preserve positivity of density and internal energy under suitable conditions.

2.1.2. Second order schemes for homogeneous equations. A standard method to obtain second order of accurate schemes for (2.2) is based on piecewise linear reconstruction using cell averages ( see $[11,19]$ ). We will follow $[18,2,3]$ and reconstruct primitive variables.

Let $u$ be a scalar function with cell average $w_{i}$ on the cell $I_{i}$. Following [18], we define a piecewise linear reconstruction function

$$
w(x)=w_{i}+D w_{i}\left(x-x_{i}\right), \quad x \in\left(x_{i-\frac{1}{2}}, x_{i+\frac{1}{2}}\right),
$$

on each cell $I_{j}$, where we need to define slop $\sigma_{i}$. We will use MinMod limiter,

$$
D w_{i}=\operatorname{minmod}\left(\frac{w_{i+1}-w_{i}}{\Delta x}, \frac{w_{i}-w_{i-1}}{\Delta x}\right),
$$

where,

$$
\operatorname{minmod}(a, b, c)=\left\{\begin{array}{l}
\operatorname{sign}(a) \min (|a|,|b|), \quad \text { if } \quad \operatorname{sign} a=\operatorname{sign} b \\
0, \quad \text { otherwise. }
\end{array}\right.
$$

The traces of linear function on boundary of the cell $I_{j}$ are,

$$
w_{i}^{ \pm}=w_{i} \pm D w_{i} \frac{\Delta x}{2},
$$

For the system (2.2), let $\hat{\mathbf{w}}_{i}$ 's are the primitive variables corresponding to the conservative variable $\mathbf{w}_{i}$. Following above reconstruction procedure component-wise, we will now define the traces, $\hat{\mathbf{w}}_{i}^{ \pm}$of $\hat{\mathbf{w}}$. We proceeds as follows: 


$$
\begin{gathered}
\rho_{\alpha, i}^{ \pm}=\rho_{\alpha, i} \pm \frac{\Delta x}{2} D \rho_{\alpha, i}, \\
\mathbf{v}_{\alpha, i}^{ \pm}=\mathbf{v}_{\alpha, i} \pm \frac{\rho_{\alpha, i}^{\mp}}{\rho_{\alpha, i}} D \mathbf{v}_{\alpha, i}, \\
\epsilon_{\alpha, i}^{ \pm}=\tilde{\epsilon}_{\alpha, i} \pm \frac{\Delta x}{2} D \epsilon_{\alpha, i},
\end{gathered}
$$

with

$$
\tilde{\epsilon}_{\alpha, i}=\epsilon_{\alpha, i}-\frac{\rho_{\alpha, i}^{+} \rho_{\alpha, i}^{-}}{\rho_{\alpha, i}} \frac{\Delta x^{2}}{8}\left|D \mathbf{v}_{\alpha, i}\right|^{2} .
$$

Let us denote $\mathbf{w}_{i}^{ \pm}$are traces of conservative variable obtained via change of variable from $\hat{\mathbf{w}}^{ \pm}$. To obtain a positive preserving reconstruction procedure we follow $[2,1,3]$ and put further conditions on the density and velocity slops,

$$
\frac{\Delta x}{2}\left|D \rho_{\alpha, i}\right|<\rho_{\alpha, i}, \quad \frac{\Delta x^{2}}{8}\left|D \mathbf{v}_{\alpha, i}\right|^{2}<\frac{\rho_{\alpha, i}}{\rho_{\alpha, i}^{+} \rho_{\alpha, i}^{-}}\left(\epsilon_{\alpha, i}-\frac{\Delta x}{2}\left|D \epsilon_{\alpha, i}\right|\right)
$$

for $\alpha \in\{i, e\}$. Under these conditions and with CFL condition of $1 / 4$, the scheme,

$$
\mathbf{w}_{i}^{n+1}=\mathbf{w}_{i}^{n}-\frac{\Delta t}{\Delta x}\left(\mathbf{F}^{x}\left(\mathbf{w}_{i}^{n,+}, \mathbf{w}_{i+1}^{n,-}\right)-\mathbf{F}^{x}\left(\mathbf{w}_{i-1}^{n,+}, \mathbf{w}_{i}^{n,-}\right)\right)
$$

is $\Omega$-invariant.

Proposition 2.2. The numerical scheme (2.20) for the Equations (2.2) with $\Omega$-invariants numerical flux preserve the positivity of density and internal energy under the usual CFL conditions $1 / 4$ if the condition (2.19) are satisifed. Furthermore, (2.20) is second order accurate in space.

Proof. See [3] for the proof.

2.2. Discretization of the source terms. In this subsection, we will present the discretization of the source terms. As discuss earlier, one of the challenge in discretization of two fluid equations is presence of highly stiff and oscillatory source terms. An efficient and robust treatment of the source is therefor necessary. This is the key contribution of this article. We will discuss discretization of the source using both explicit and implicit schemes.

Let us consider the source ordinary differential equation,

$$
\frac{d \mathbf{w}_{i}}{d t}=\mathbf{s}\left(\mathbf{w}_{i}\right) .
$$

Assume that initial data $\mathbf{w}_{i}^{n}$, is in $\Omega$, i.e.

$$
\rho_{\alpha, i}^{n}>0 \quad \text { and } \quad \epsilon_{\alpha, i}^{n}>0, \quad \alpha \in\{i, e\} .
$$

We are interested in the discretization of $(2.21)$, so that $\mathbf{w}_{i}^{n+1}$ is in $\Omega$. We first consider explicit scheme.

2.2.1. Explicit scheme. We consider the explicit discretization of (2.21). Using a standard forward Euler scheme, we get,

$$
\mathbf{w}_{i}^{n+1}=\mathbf{w}_{i}^{n}+\Delta t \mathbf{s}\left(\mathbf{w}_{i}^{n}\right)
$$

We will denote this update by,

$$
\mathbf{w}_{i}^{n+1}=\mathcal{S}_{\Delta t}^{e, 1} \mathbf{w}_{i}^{n}
$$


Note that,

$$
\rho_{\alpha, i}^{n+1}=\rho_{\alpha, i}^{n} \quad \alpha \in\{i, e\}
$$

So, positivity of density is trivial. We will now consider positivity of internal energy. The momentum update is,

$$
\rho_{\alpha, i}^{n+1} \mathbf{v}_{\alpha, i}^{n+1}=\rho_{\alpha, i}^{n} \mathbf{v}_{\alpha, i}^{n}+\Delta t \frac{\rho_{\alpha, i}^{n}}{\hat{l}_{r}^{\alpha}}\left(\mathbf{E}_{i}^{n}+\mathbf{v}_{\alpha, i}^{n} \times \mathbf{B}_{i}^{n}\right),
$$

where $\hat{l}_{r}^{\alpha}=\hat{l}_{r}$, if $\alpha=i$ and $\hat{l}_{r}^{\alpha}=-\frac{\hat{l}_{r}}{m}$, if $\alpha=e$. This implies,

$$
\mathbf{v}_{\alpha, i}^{n+1}=\mathbf{v}_{\alpha, i}^{n}+\Delta t \frac{1}{\hat{l}_{r}^{\alpha}}\left(\mathbf{E}_{i}^{n}+\mathbf{v}_{\alpha, i}^{n} \times \mathbf{B}_{i}^{n}\right) .
$$

The internal energy at time $t^{n}+\Delta t$ is,

$$
\begin{gathered}
\epsilon_{\alpha, i}^{n+1}=\mathcal{E}_{\alpha, i}^{n+1}-\frac{1}{2} \rho_{\alpha, i}^{n+1}\left|\mathbf{v}_{\alpha, i}^{n+1}\right|^{2} \\
=\mathcal{E}_{\alpha, i}^{n}+\Delta t \frac{\rho_{\alpha, i}^{n}}{\hat{l}_{r}^{\alpha}}\left(\mathbf{E}_{i}^{n} \cdot \mathbf{v}_{\alpha, i}^{n}\right)-\frac{1}{2} \rho_{\alpha, i}^{n+1}\left(\left|\mathbf{v}_{\alpha, i}^{n}\right|^{2}+2 \frac{\Delta t}{\hat{l}_{r}^{\alpha}} \mathbf{v}_{\alpha, i}^{n} \cdot\left(\mathbf{E}_{i}^{n}+\mathbf{v}_{\alpha, i}^{n} \times \mathbf{B}_{i}^{n}\right)\right. \\
\left.+\left(\frac{\Delta t}{\hat{l_{r}^{\alpha}}}\right)^{2}\left|\left(\mathbf{E}_{i}^{n}+\mathbf{v}_{\alpha, i}^{n} \times \mathbf{B}_{i}^{n}\right)\right|^{2}\right) \\
=\mathcal{E}_{\alpha, i}^{n}-\frac{1}{2} \rho_{\alpha, i}^{n}\left|\mathbf{v}_{\alpha, i}^{n}\right|^{2}-\frac{\Delta t^{2}}{2\left|\hat{l}_{r}^{\alpha}\right|^{2}} \rho_{\alpha, i}^{n}\left|\left(\mathbf{E}_{i}^{n}+\mathbf{v}_{\alpha, i}^{n} \times \mathbf{B}_{i}^{n}\right)\right|^{2} \\
=\epsilon_{\alpha, i}^{n}-\frac{\Delta t^{2}}{2\left|\hat{l}_{r}^{\alpha}\right|^{2}} \rho_{\alpha, i}^{n}\left|\left(\mathbf{E}_{i}^{n}+\mathbf{v}_{\alpha, i}^{n} \times \mathbf{B}_{i}^{n}\right)\right|^{2}
\end{gathered}
$$

So, for internal energy to be positive we need,

i.e.

$$
\epsilon_{\alpha, i}^{n}>\frac{\Delta t^{2}}{2\left|\hat{l}_{r}^{\alpha}\right|^{2}} \rho_{\alpha, i}^{n}\left|\left(\mathbf{E}_{i}^{n}+\mathbf{v}_{\alpha, i}^{n} \times \mathbf{B}_{i}^{n}\right)\right|^{2}
$$

$$
\begin{gathered}
\Delta t<\sqrt{2\left|\hat{l}_{r}^{\alpha}\right|^{2} \frac{\epsilon_{\alpha, i}^{n}}{\rho_{\alpha, i}^{n}\left|\left(\mathbf{E}_{i}^{n}+\mathbf{v}_{\alpha, i}^{n} \times \mathbf{B}_{i}^{n}\right)\right|^{2}}}, \\
\Delta t<\sqrt{\frac{\epsilon_{\alpha, i}^{n}}{\rho_{\alpha, i}^{n}} \frac{\sqrt{2}\left|\hat{l}_{r}^{\alpha}\right|}{\left|\left(\mathbf{E}_{i}^{n}+\mathbf{v}_{\alpha, i}^{n} \times \mathbf{B}_{i}^{n}\right)\right|}} .
\end{gathered}
$$

With this restriction on time step, forward Euler scheme update (2.24) is $\Omega$-invariant.

Theorem 2.3. The source update (2.24) is $\Omega$-invariant if time step satisfies (2.25) for $\alpha \in\{i, e\}$.

The analysis above provide exact restriction on the time step for the explicit euler update to be $\Omega$-invariant. The time step depend on Larmor radius, internal energy, density and electromagnetic force. Note that as Larmor radius decreases, source terms becomes for stiff and we have to pick small enough time step. In addition, in the case of low internal energy ( or low pressure), we need time step to be small. Also, the error in this case decreases internal energy of the system.

Now we will consider the implicit scheme. 
2.2.2. Implicit scheme. The backward Euler scheme for (2.21) is,

$$
\mathbf{w}_{i}^{n+1}=\mathbf{w}_{i}^{n}+\Delta t \mathbf{s}\left(\mathbf{w}_{i}^{n+1}\right) .
$$

We need to solve the Equation (2.26) for $\mathbf{w}_{i}^{n+1}$ to update the solution. We proceed as follows: Let us divide $\mathbf{w}$ in the following components,

$$
\begin{aligned}
\mathbf{w}^{a} & =\left\{\rho_{i}, \rho_{e}, \psi, \mathbf{B}\right\}^{\top}, \\
\mathbf{w}^{b} & =\left\{\rho_{i} \mathbf{v}_{i}, \rho_{e} \mathbf{v}_{e}, \mathbf{E}\right\}^{\top}, \\
\mathbf{w}^{c} & =\left\{\mathcal{E}_{i}, \mathcal{E}_{e}, \phi\right\}^{\top},
\end{aligned}
$$

then we can rewrite $(2.26)$ as,

$$
\begin{aligned}
\mathbf{w}_{i}^{a, n+1} & =\mathbf{w}_{i}^{a, n}, \\
\mathbf{w}_{i}^{b, n+1} & =\mathbf{w}_{i}^{b, n}+\Delta t \mathcal{A}\left(\mathbf{w}_{i}^{a, n+1}\right) \mathbf{w}_{i}^{b, n+1}, \\
\mathbf{w}_{i}^{c, n+1} & =\mathbf{w}_{i}^{c, n}+\mathbf{G}\left(\mathbf{w}_{i}^{a, n+1}, \mathbf{w}_{i}^{b, n+1}\right) .
\end{aligned}
$$

To update $\mathbf{w}_{i}^{n+1}$, we first needs to solve $(2.28 \mathrm{~b})$, which is linear with matrix $\mathcal{A}\left(\mathbf{w}^{a, n+1}\right)$ is given by,

$$
\left[\begin{array}{ccc}
0 & \frac{B^{z,(n+1)}}{\hat{r} g} & -\frac{B^{y,(n+1)}}{\hat{r} g} \\
-\frac{B^{z,(n+1)}}{\hat{r} g} & 0 & \frac{B^{x,(n+1)}}{\hat{r} g} \\
\frac{B^{y,(n+1)}}{\hat{r} g} & -\frac{B^{x,(n+1)}}{\hat{r} g} & 0 \\
0 & 0 & 0 \\
0 & 0 & 0 \\
0 & 0 & 0 \\
\frac{-r_{i}}{L} & 0 & 0 \\
0 & \frac{-r_{i}}{L} & 0 \\
0 & 0 & \frac{-r_{i}}{L}
\end{array}\right.
$$
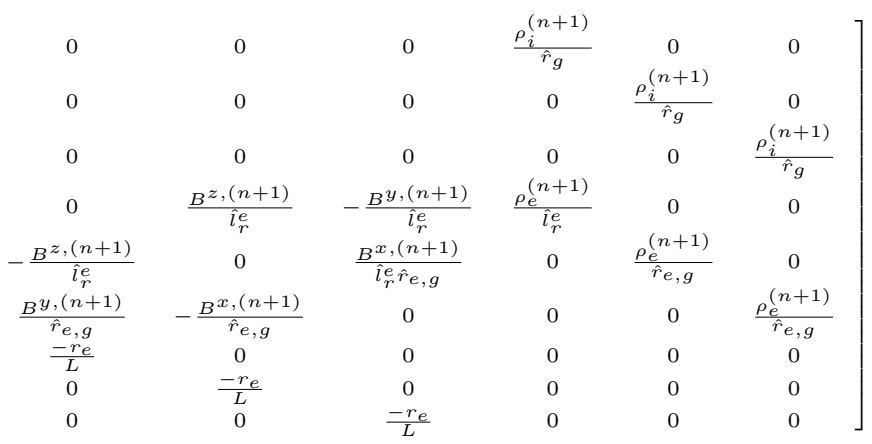

Here $\hat{l}_{r}^{e}=-\hat{l}_{r} / m$ and $L=\hat{\lambda}_{d}^{2} \hat{l}_{r}$. So, we can solved it either exactly, or using any standard linear solver (see [12]). The update $\mathbf{w}_{i}^{c, n+1}$ can now be easily evaluated as $\mathbf{G}$ ( which represent the source term of ion and electron energies and potential $\phi$ ) depend on $\mathbf{w}_{i}^{a, n+1}$ and $\mathbf{w}_{i}^{b, n+1}$ only. This is because source term of energies and potential $\phi$ depends on $\mathbf{w}^{a}$ and $\mathbf{w}^{b}$, not on $\mathbf{w}^{c}$.

We will denote this update as,

$$
\mathbf{w}_{i}^{n+1}=\mathcal{S}_{\Delta t}^{i, 1} \mathbf{w}_{i}^{n} .
$$

We will now analyze the $\Omega$-invariance of this update. As in the case of explicit equations,

$$
\rho_{\alpha, i}^{n+1}=\rho_{\alpha, i}^{n} .
$$

Also,

$$
\rho_{\alpha, i}^{n+1} \mathbf{v}_{\alpha, i}^{n+1}=\rho_{\alpha, i}^{n} \mathbf{v}_{\alpha, i}^{n}+\Delta t \frac{\rho_{\alpha, i}^{n+1}}{\hat{l}_{r}^{\alpha}}\left(\mathbf{E}_{i}^{n+1}+\mathbf{v}_{\alpha, i}^{n+1} \times \mathbf{B}_{i}^{n+1}\right) .
$$

Combining it with density update,

$$
\mathbf{v}_{\alpha, i}^{n+1}=\mathbf{v}_{\alpha, i}^{n}+\Delta t \frac{1}{\hat{l}_{r}^{\alpha}}\left(\mathbf{E}_{i}^{n+1}+\mathbf{v}_{\alpha, i}^{n+1} \times \mathbf{B}_{i}^{n+1}\right) .
$$

Now internal energy at $t^{n}+\Delta t$ is,

$$
\epsilon_{\alpha, i}^{n+1}=\mathcal{E}_{\alpha, i}^{n+1}-\frac{1}{2} \rho_{\alpha, i}^{n+1}\left|\mathbf{v}_{\alpha, i}^{n+1}\right|^{2}
$$




$$
\begin{gathered}
=\mathcal{E}_{\alpha, i}^{n}+\Delta t \frac{\rho_{\alpha, i}^{n+1}}{\hat{l}_{r}^{\alpha}}\left(\mathbf{E}_{i}^{n+1} \cdot \mathbf{v}_{\alpha, i}^{n+1}\right)-\frac{1}{2} \rho_{\alpha, i}^{n+1}\left(\left|\mathbf{v}_{\alpha, i}^{n}\right|^{2}+2 \frac{\Delta t}{\hat{l}_{r}^{\alpha}} \mathbf{v}_{\alpha, i}^{n} \cdot\left(\mathbf{E}_{i}^{n+1}+\mathbf{v}_{\alpha, i}^{n+1} \times \mathbf{B}_{i}^{n+1}\right)\right. \\
\left.+\left(\frac{(\Delta t)^{2}}{\left|\hat{l}_{r}^{\alpha}\right|^{2}}\right)\left|\left(\mathbf{E}_{i}^{n+1}+\mathbf{v}_{\alpha, i}^{n+1} \times \mathbf{B}_{i}^{n+1}\right)\right|^{2}\right) \\
=\epsilon_{\alpha, i}^{n}+\frac{\Delta t}{\hat{l}_{r}^{\alpha}} \rho_{\alpha, i}^{n+1}\left(\mathbf{v}_{\alpha, i}^{n+1} \cdot\left(\mathbf{E}_{i}^{n+1}+\mathbf{v}_{\alpha, i}^{n+1} \times \mathbf{B}_{i}^{n+1}\right)-\mathbf{v}_{\alpha, i}^{n} \cdot\left(\mathbf{E}_{i}^{n+1}+\mathbf{v}_{\alpha, i}^{n+1} \times \mathbf{B}_{i}^{n+1}\right)\right) \\
-\frac{\rho_{\alpha, i}^{n+1}(\Delta t)^{2}}{2\left|\hat{l}_{r}^{\alpha}\right|^{2}}\left|\left(\mathbf{E}_{i}^{n+1}+\mathbf{v}_{\alpha, i}^{n+1} \times \mathbf{B}_{i}^{n+1}\right)\right|^{2} \\
=\epsilon_{\alpha, i}^{n}+\frac{\Delta t}{\hat{l}_{r}^{\alpha}} \rho_{\alpha, i}^{n+1}\left(\left(\mathbf{v}_{\alpha, i}^{n+1}-\mathbf{v}_{\alpha, i}^{n}\right) \cdot\left(\mathbf{E}_{i}^{n+1}+\mathbf{v}_{\alpha, i}^{n+1} \times \mathbf{B}_{i}^{n+1}\right)\right)-\frac{\rho_{\alpha, i}^{n+1}(\Delta t)^{2}}{2\left|\hat{l}_{r}^{\alpha}\right|^{2}}\left|\left(\mathbf{E}_{i}^{n+1}+\mathbf{v}_{\alpha, i}^{n+1} \times \mathbf{B}_{i}^{n+1}\right)\right|^{2} \\
=\epsilon_{\alpha, i}^{n}+\frac{\rho_{\alpha, i}^{n+1}(\Delta t)^{2}}{\left|\hat{l}_{r}^{\alpha}\right|^{2}}\left|\left(\mathbf{E}_{i}^{n+1}+\mathbf{v}_{\alpha, i}^{n+1} \times \mathbf{B}_{i}^{n+1}\right)\right|^{2}-\frac{\rho_{\alpha, i}^{n+1}(\Delta t)^{2}}{2\left|\hat{l}_{r}^{\alpha}\right|^{2}}\left|\left(\mathbf{E}_{i}^{n+1}+\mathbf{v}_{\alpha, i}^{n+1} \times \mathbf{B}_{i}^{n+1}\right)\right|^{2} \\
=\epsilon_{\alpha, i}^{n}+\frac{\rho_{\alpha, i}^{n}(\Delta t)^{2}}{2\left|\hat{l}_{r}^{\alpha}\right|^{2}}\left|\left(\mathbf{E}_{i}^{n+1}+\mathbf{v}_{\alpha, i}^{n+1} \times \mathbf{B}_{i}^{n+1}\right)\right|^{2}
\end{gathered}
$$

So, internal energy is unconditionally positive. Hence, implicit step is unconditionally $\Omega$-invariant.

Theorem 2.4. The source update (2.30) is $\Omega$-invariant for any $\Delta t>0$.

The analysis for the implicit scheme provide the exact relation between internal energy before and after the update (2.30). We observe that internal energy is actually increased by this step. So, we don't need any restriction on time step for the update to be $\Omega$-invariant. Furthermore, note that the update is increasing the pressure and density is unchanged. So, the implicit source update is also entropy stable. This is not the case for the explicit update (2.30).

2.3. Discretization of two fluid equations. First order schemes for (1.3) can be obtained by combining first order numerical schemes for homogeneous part (2.4) with source terms discretizations (2.24) and (2.26) via Godunov's splitting. In the case of explicit scheme we get,

$$
\mathbf{w}_{i}^{n+1}=\mathcal{S}_{\Delta t}^{e, 1} \mathcal{H}_{\Delta t}^{1} \mathbf{w}_{i}^{n} .
$$

This scheme will be $\Omega$-invariant under usual CFL condition, if $\Delta t$ satisfies (2.25) for the intermediate update $\mathcal{H}_{\Delta t}^{1} \mathbf{w}_{i}^{n}$ (see Theorem 2.3).

If source treated implicitly, we have,

$$
\mathbf{w}_{i}^{n+1}=\mathcal{S}_{\Delta t}^{i, 1} \mathcal{H}_{\Delta t}^{1} \mathbf{w}_{i}^{n} .
$$

The scheme will be $\Omega$-invariant under usual CFL condition as source update is unconditionally $\Omega$-invariant (see Theorem 2.4).

Remark 2.5. In the case of explicit schemes, the condition (2.25) has to be satisfied by $\mathcal{H}_{\Delta t}^{1} \mathbf{w}_{i}^{n}$, whereas we have already chosen $\Delta t$ to calculate $\mathcal{H}_{\Delta t}^{1} \mathbf{w}_{i}^{n}$ and source update. To overcome this difficulty, we put restriction (2.25) on $\mathbf{w}_{i}^{n}$ and reduce $\Delta t$ slightly by choosing, $\Delta t=\Delta t-1 / 5 \Delta t$. Note that we still check that new $\Delta t$ satisfies (2.25) for $\mathcal{H}_{\Delta t}^{1} \mathbf{w}_{i}^{n}$. If this condition is violated then we choose smaller $\Delta t$ and again calculate $\mathcal{H}_{\Delta t}^{1} \mathbf{w}_{i}^{n}$. We remark that the solution will note change drastically in one time step. So, the $\Delta t$ chosen above is usually sufficient.

2.3.1. Second order schemes. To obtain second order source discretization we will use SSP RungeKutta schemes (see [16]) upto third order. We replace each intermediate Euler step with a first order update. As the final update is linear combination of intermediate states with positive coefficients, the final update will also be $\Omega$-invariant. We will then use combine homogeneous part with source using Strang splitting. 
Explicit schemes. Let us denote second order explicit source update with $\mathcal{S}_{\Delta t}^{e, 2}$ and third order explicit source update with $\mathcal{S}_{\Delta t}^{e, 3}$. Similarly, let $\mathcal{H}_{\Delta t}^{2}$ denotes update using second order RungeKutta method and $\mathcal{H}_{\Delta t}^{3}$ denotes update using third order Runge-Kutta method with each euler update is obtained by (2.20). We make sure that each intermediate step satisfies $(2.25)$ by choosing time step small enough (see Remark 2.5).

Using the Strang splitting we consider two second order explicit scheme:

- O2-222-exp scheme

- O2-333-exp scheme

$$
\mathbf{w}_{i}^{n+1}=\mathcal{S}_{\frac{\Delta t}{2}}^{e, 2} \mathcal{H}_{\Delta t}^{2} \mathcal{S}_{\frac{\Delta t}{2}}^{e, 2} \mathbf{w}_{i}^{n}
$$

$$
\mathbf{w}_{i}^{n+1}=\mathcal{S}_{\frac{\Delta t}{2}}^{e, 3} \mathcal{H}_{\Delta t}^{3} \mathcal{S}_{\frac{\Delta t}{2}}^{e, 3} \mathbf{w}_{i}^{n}
$$

We have,

Theorem 2.6. The second order schemes (2.33) and (2.34) are $\Omega$ invariant with CFL $1 / 4$ if intermediate states satisfies there respective time step condition.

Implicit-Explicit schemes. Similar to the explicit case we consider second order implicit source updates $\mathcal{S}_{\Delta t}^{i, 2}$ based on RK2 and third order implicit source update, $\mathcal{S}_{\Delta t}^{i, 3}$ based on RK3, with euler step replace by, (2.26). Combining, the homogeneous and source parts via Strang splitting we consider following schemes:

- O2-222-imex scheme

- O2-333-imex scheme

$$
\mathbf{w}_{i}^{n+1}=\mathcal{S}_{\frac{\Delta t}{2}}^{i, 2} \mathcal{H}_{\Delta t}^{2} \mathcal{S}_{\frac{\Delta t}{2}}^{i, 2} \mathbf{w}_{i}^{n}
$$

$$
\mathbf{w}_{i}^{n+1}=\mathcal{S}_{\frac{\Delta t}{2}}^{i, 3} \mathcal{H}_{\Delta t}^{3} \mathcal{S}_{\frac{\Delta t}{2}}^{i, 3} \mathbf{w}_{i}^{n}
$$

These scheme are robust in the following sense:

Theorem 2.7. The second order numerical schemes (2.35) and (2.36) are $\Omega$ invariant if homogeneous operators are $\Omega$ invariant.

\section{Numerical EXPERIMENTS}

In this Section, we consider three numerical examples to demonstrated the accuracy, robustness and efficiency of the schemes presented so far.

3.1. Convergence rates. The first numerical experiment is used to check the numerical order of accuracy of the schemes. We set initial and boundary conditions, in such a way that we have exact solution of two fluid equations. We consider the domain $[0,1]$ with periodic boundary conditions. Initial densities are chosen to be $\rho_{i}=\rho_{e}=2.0+\sin (2 \pi x)$. We consider the flow with constant velocities $v_{i}^{x}=v_{e}^{x}=1.0$ in $x$-direction, at constant pressure $p_{i}=p_{e}=1.0$. We take initial magnetic field $B^{y}=\sin (2 \pi x)$ and electric field $E^{z}=-\sin 2 \pi x$. All other components of velocities, magnetic field and electric field are set to be zero. If we take mass ratio $m=1.0$ then, these conditions will give us the exact solution,

$$
\rho_{i}=\rho_{e}=2.0+\sin (2 \pi(x-t)) .
$$

However, with the mass ratio of 1.0 , source terms will be zero. To avoid this situation, we consider mass ratio to be 2.0 and compensate by modifying the equations. So, instead of (1.3), we will simulate,

$$
\mathbf{u}_{t}+\nabla \cdot \mathbf{f}(\mathbf{u})=\mathbf{s}(\mathbf{u})+R(x, t) .
$$



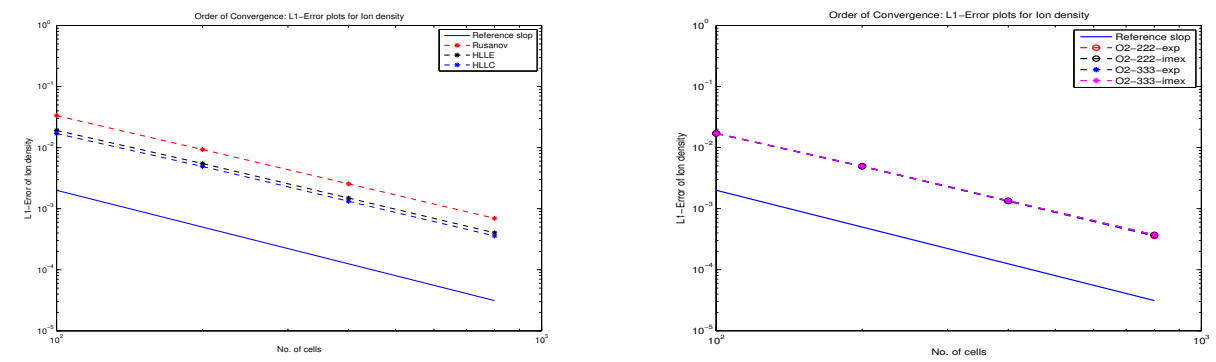

(a) Convergence Rates: Comparison of HLLC, (b) Convergence Rates: Comparison of O2-222HLLE and Rusanov solvers using O2-333-exp exp, O2-222-imex, O2-333-exp and O2-333-imex scheme. schemes using HLLC solvers

Figure 1. Rate of convergence plots

where $R(x, t)$ is,

$$
\left.\left.\left\{\mathbf{0}_{13},-(2+\sin 2 \pi(x-t))\right), 0,0,2+\sin 2 \pi(x-t)\right), 0\right\}^{\top} .
$$

Numerical rates of convergence for $L^{1}$ errors for ion-density are plotted in Figure 1. In Figure 1(a) we have compared rates of convergence for different solvers using O2-333-exp scheme. We observe that all the solvers have second order of convergence. In addition, the Rusanov solver is most diffusive, followed by HLLE and HLLC solver. We conclude that HLLC solver is most accurate, which was expected.

In Figure 1(b), we have used HLLC solver and compared the L1-errors of time stepping methods O2-222-exp, O2-222-imex, O2-333-exp and O2-333-imex. All these methods have similar errors and have second order of convergence.

3.2. Soliton Propagation. In two-fluid plasma, evolution and interaction of solitons is simulated in $[12,4,6,5,9]$. In these articles, an initial density hump is used produce ion-acoustics solitons. Following [12], we consider an initial ion density to be,

$$
\rho_{i}=1.0+\exp (-25.0|x-L / 3|),
$$

with $L=12$. The electron pressure is taken to be $p_{e}=5 \rho_{i}$. The ion pressure is $1 / 100$ th of electron pressure. We consider mass-ratio $m=25.0$. The computational domain considered is $D=(0, L)$ with periodic boundary conditions. Initially, plasma is at rest and all electromagnetic quantities are considered to be zero. Normalized Debye length is taken to be 1.0. We will consider Larmor radii of $0.01,0.0001$ and $10^{-6}$, in increasing order of stiffness of the source. All the simulations presented in this subsection are simulated on 8 cores.

First, we will compare different numerical fluxes. In Figure 2, we have plotted the results for HLLC, HLLE and Rusanov solvers with $\hat{l}_{r}=0.01$ using O2-333-imex scheme on 3000 cells. In Figure 2(a), we have plotted the evolution of ion-density at non-dimensional time $t=1,2,3,4$ and 5. All the solvers captures soliton formation and there nonlinear interactions, successfully. In Figure 2(b), we have plotted the ion-density at $t=5.0$. We have also plotted the reference solution which was computed using 20000 cells. We observe that HLLC solvers is more accurate then HLLE and Rusanov solvers. HLLE and Rusanov solver have similar accuracy. This is further evident from Figure 2(c) where we have zoomed in at $x=4.0$.

When compared to simulation performed in $[12,9]$, we note that our schemes are more diffusive. In fact, both wave propagation algorithm of [9] and entropy stable schemes of [12] are better at capturing small scale oscillations. However, we are using MinMod limiter compared 

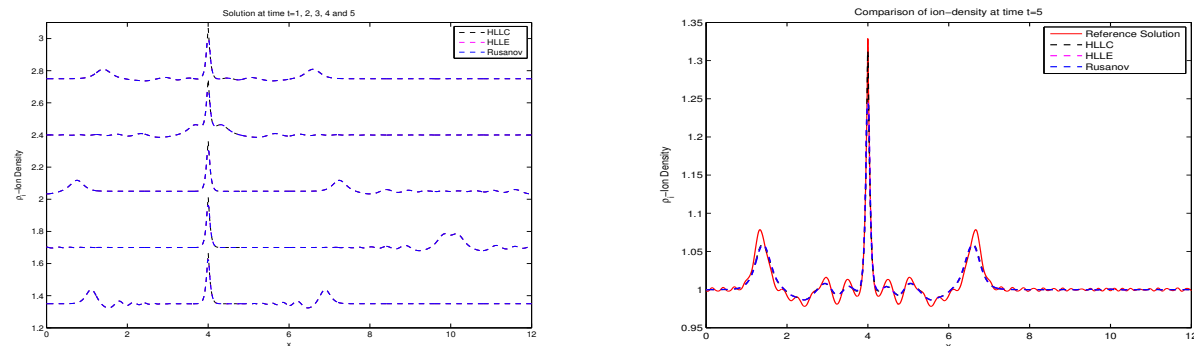

(a) Ion density at non-dimensional time of $t=(\mathrm{b})$ Ion density at non-dimensional time of $t=5$ 1, 2,3, 4 and 5 using HLLC, HLLE and Rusanov using HLLC, HLLE and Rusanov solvers. Refersolvers. ence solution is also plotted for comparison.

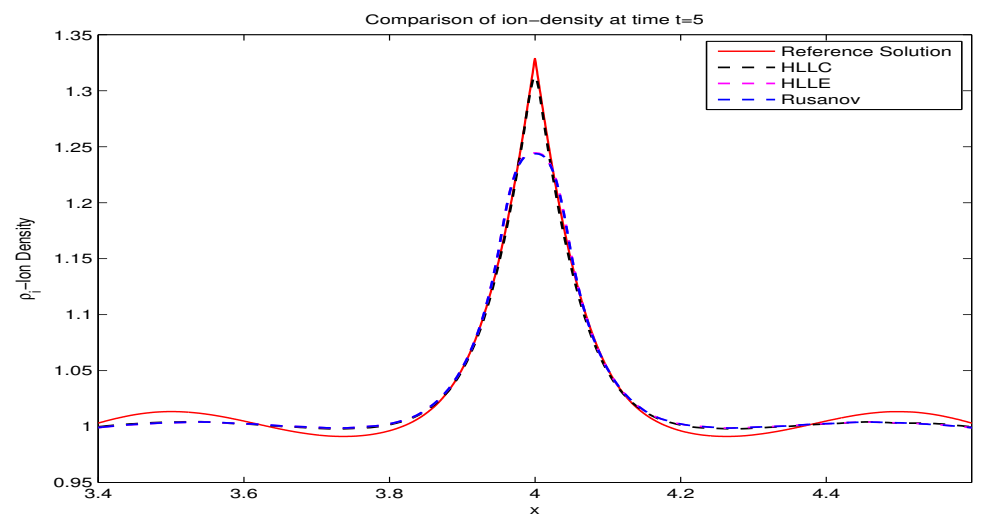

(c) Ion density at non dimensional time $t=5.0$ using HLLC, HLLE and Rusanov solvers. Zoomed at $x=4.0$. Reference solution is also plotted.

Figure 2. Soliton Propagation: Evolution of solitons with Larmor radius of 0.01 using 3000 cells and O2-333-imex time stepping method. We compare results with HLLC, HLLE and Rusanov numerical fluxes. Reference solution was computed using HLLC solver with o2-333-imex scheme on 20000 cells.

to the MC limiter of [9]. Also, [9] uses fourth order time-stepping to resolve source effects. We would also like to point that the solutions with HLLC solvers is on par with [9] and better then $[12]$ at capturing the stationary hump at $x=4.0$.

In Figures 3 and 4, we have plotted results for $\hat{l}_{r}=10^{-4}$ and $\hat{l}_{r}=10^{-6}$ respectively. Again from Figures 3(a) and 4(a), we observe that all the solvers capture solution waves and there interactions accurately. Furthermore, HLLC solver is more accurate then the HLLC and Rusanov solvers (see Figs. 3(b) and 4(b)).

As HLLC solver found to be most accurate, in the following simulation we will use it to compare our time-stepping schemes, namely, O2-222-exp, O2-222-imex, O2-333-exp and O2-333imex. We will again consider three different values of $\hat{l}_{r}, 10^{-2}, 10^{-4}$ and $10^{-6}$.

Numerical results for the case of $10^{-2}$ are presented in Figure 5. We use HLLC solvers on 3000 cells and compare the results for O2-222-exp, O2-222-imex, O2-333-exp and O2-333imex schemes. In Figure 5(a), we note that all the schemes captures the soliton interactions. Furthermore, solutions of all the schemes are comparable (see Figure 5(b)). After zooming at 

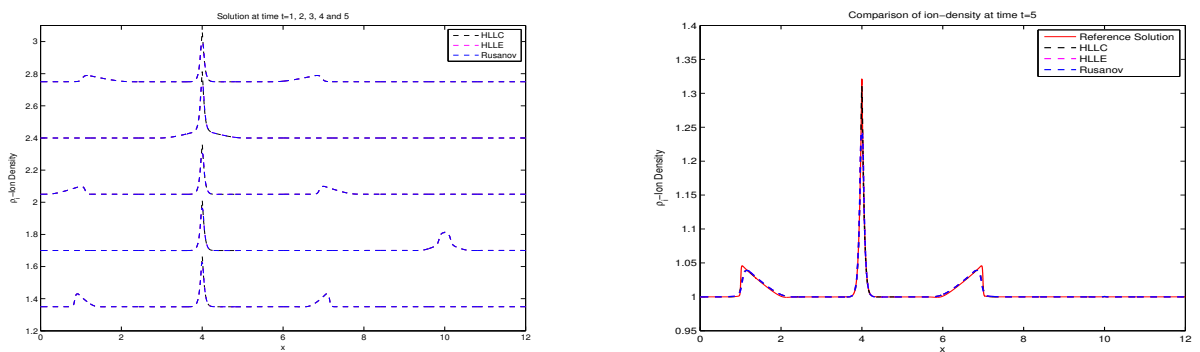

(a) Ion density at non-dimensional time of $t=(\mathrm{b})$ Ion density at non-dimensional time of $t=5$ $1,2,3,4$ and 5 using HLLC, HLLE and Rusanov using HLLC, HLLE and Rusanov solvers. Refersolvers. ence solution is also plotted for comparison.

FIGURE 3. Soliton Propagation: Evolution of solitons with Larmor radius of 0.0001 using 3000 cells and O2-333-imex time stepping method. We compare results with HLLC, HLLE and Rusanov numerical fluxes. Reference solution was computed using HLLC solver with o2-333-imex scheme on 20000 cells.
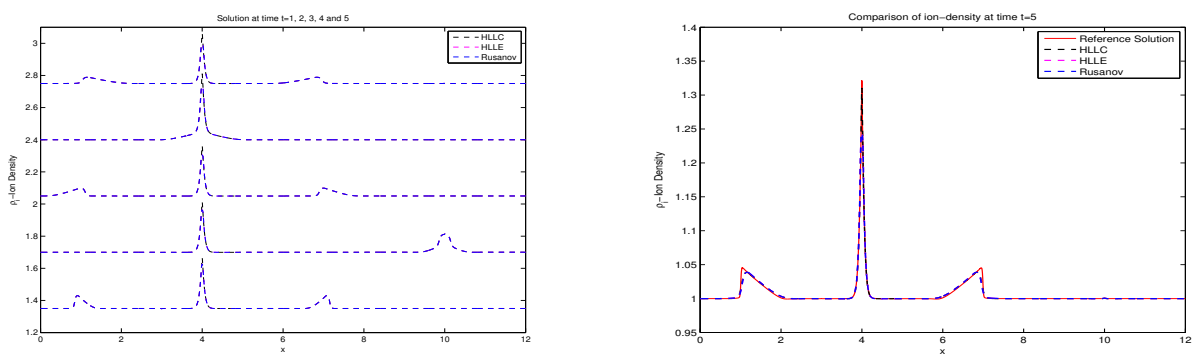

(a) Ion density at non-dimensional time of $t=$ (b) Ion density at non-dimensional time of $t=5$ 1, 2,3,4 and 5 using HLLC, HLLE and Rusanov using HLLC, HLLE and Rusanov solvers. Refersolvers. ence solution is also plotted for comparison.

Figure 4. Soliton Propagation: Evolution of solitons with Larmor radius of $10^{-6}$ using 3000 cells and O2-333-imex time stepping method. We compare results with HLLC, HLLE and Rusanov numerical fluxes. Reference solution was computed using HLLC solver with o2-333-imex scheme on 20000 cells.

$x=4.0$ we note that explicit schemes are slightly less diffusive than IMEX schemes. There is no difference in O2-222-exp and O2-333-exp schemes. Similarly, solutions of O2-222-imex and O2-333-imex schemes are on the top of each others.

Similar results are obtained for the case with $\hat{l}_{r}=10^{-4}$ (see Figure 6). However, when we consider $\hat{l}_{r}=10^{-6}$, it is not possible to compute for the explicit schemes. So, we have plotted results for IMEX schemes only (see Figure 7). The results also compare well with the corresponding simulations in [12].

The interesting part of these comparisons is simulation time of the schemes. Note that, as Larmor radius $\hat{l}_{r}$ decreases, stiffness of source increases and IMEX schemes become more efficient than explicit schemes. In Table 1, we have presented simulation times of each schemes. At $\hat{l}_{r}=10^{-2}$ the source is not stiff. So, simulation time of explicit and IMEX schemes is similar. 

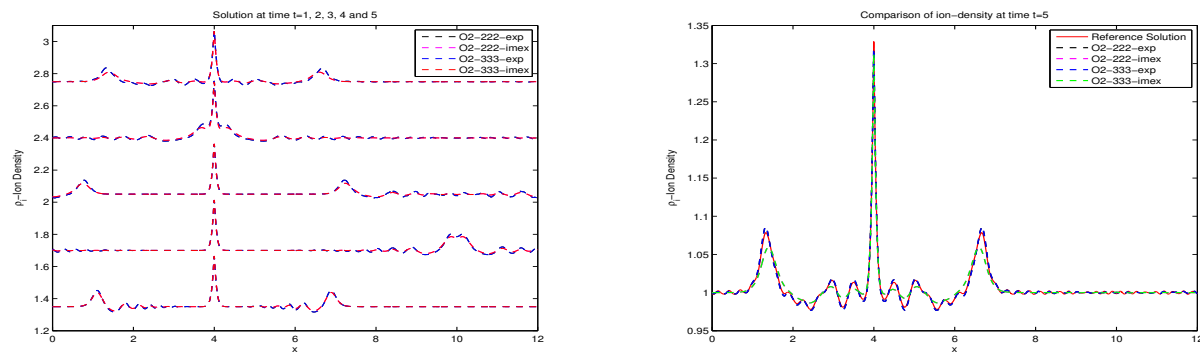

(a) Ion density at non-dimensional time of $t=$ (b) Ion density at non-dimensional time of $t=5$ 1, 2, 3, 4 and 5 using O2-222-exp, O2-222-imex, O2- using O2-222-exp, O2-222-imex, O2-333-exp and 333-exp and O2-333-imex schemes. O2-333-imex schemes. Reference solution is also plotted for comparison.

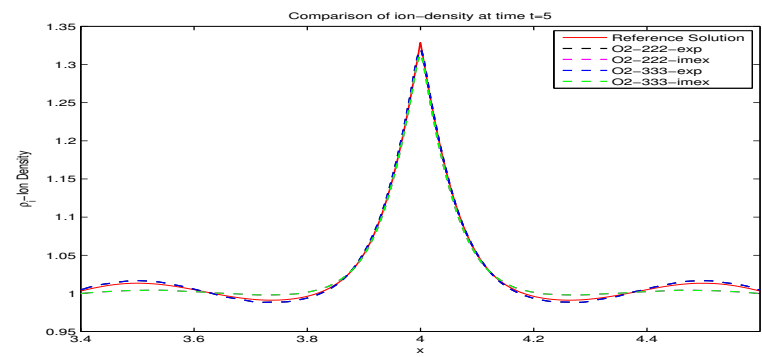

(c) Ion density at non-dimensional time of $t=5$ using O2-222-exp, O2222-imex, O2-333-exp and O2-333-imex schemes. Zoomed at $x=4.0$

Figure 5. Soliton Propagation: Evolution of solitons with Larmor radius of $10^{-2}$ using 3000 cells and HLLC solver. We compare results for O2-222-exp, O2-222-imex, O2-333-exp and O2-333-imex time stepping. Reference solution was computed using HLLC solver with o2-333-imex scheme on 20000 cells.

\begin{tabular}{|c|c|c|c|}
\hline$\hat{l}_{r}$ & $10^{-2}$ & $10^{-4}$ & $10^{-6}$ \\
\hline O2-222-exp & 140.9 & 2283.2 & - \\
\hline O2-222-imex & 134.5 & 133.9 & 134.9 \\
\hline O2-333-exp & 214.3 & 208.3 & - \\
\hline O2-333-imex & 202.5 & 201.9 & 201.1 \\
\hline
\end{tabular}

TABLE 1. Simulation time of soliton evolution with different schemes

At $\hat{l}_{r}=10^{-4}$ stiffness has increase. We note that O2-222-exp scheme is highly inefficient, whereas all other schemes take similar simulation time. We observe that O2-333-exp is more efficient than O2-222-exp. At $\hat{l}_{r}=10^{-6}$ both explicit schemes are highly inefficient. In fact, we were not able to commute solution using them. On the other hand IMEX schemes take same computational time for all the values of $\hat{l}_{r}$. This shows the efficiency of the IMEX schemes with respect to the stiff source.

As we have already seen that HLLC solver is most accurate, we will only use HLLC solvers in next Sections. Furthermore, we will also consider only O2-333-exp and O2-333-imex schemes for 

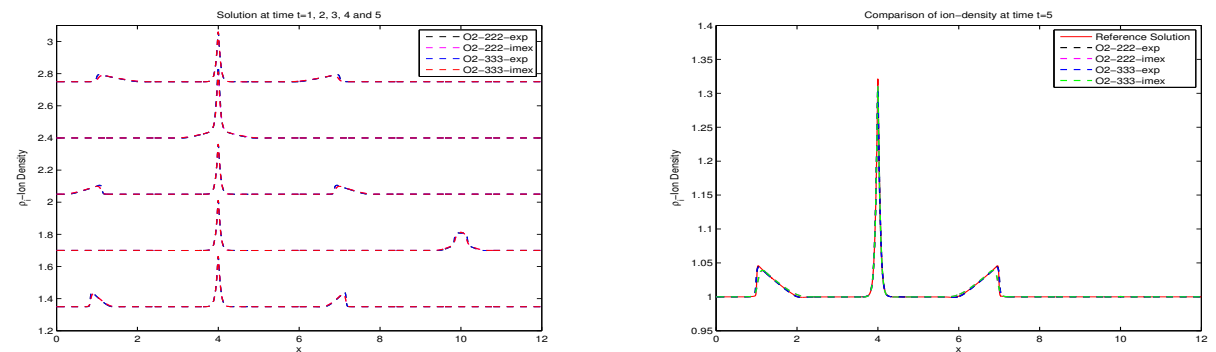

(a) Ion density at non-dimensional time of $t=$ (b) Ion density at non-dimensional time of $t=5$ 1, 2, 3, 4 and 5 using O2-222-exp, O2-222-imex, O2- using O2-222-exp, O2-222-imex, O2-333-exp and 333-exp and O2-333-imex schemes. O2-333-imex schemes. Reference solution is also plotted for comparison.

Figure 6. Soliton Propagation: Evolution of solitons with Larmor radius of $10^{-4}$ using 3000 cells and HLLC solver. We compare results for O2-222-exp, O2-222-imex, O2-333-exp and O2-333-imex time stepping. Reference solution was computed using HLLC solver with o2-333-imex scheme on 20000 cells.
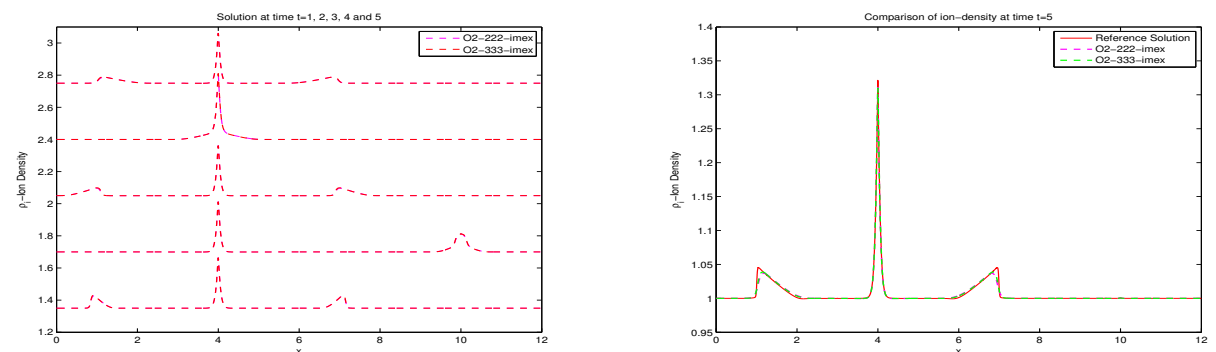

(a) Ion-density at non-dimensional time of $t=(\mathrm{b})$ Ion-density at non-dimensional time of $t=5$ 1,2,3, 4 and 5 using O2-222-exp, O2-222-imex, O2- using O2-222-exp, O2-222-imex, O2-333-exp and 333-exp and O2-333-imex schemes. O2-333-imex schemes. Reference solution is also plotted for comparison.

Figure 7. Soliton Propagation: Evolution of solitons with Larmor radius of $10^{-6}$ using 3000 cells and HLLC solver. We compare results for O2-333-exp and O2-333-imex time stepping. Reference solution was computed using HLLC solver with o2-333-imex scheme on 20000 cells.

computations, to provide fare comparison between explicit and IMEX schemes, as O2-222-exp is very inefficient.

3.3. Soliton Propagation in 2D. Soliton formation in two dimension is simulated in [5, 12]. These simulations are generalization of simulations presented in previous Section. Following [12], we consider the initial ion-density profile,

$$
\rho_{i}=1+5 \exp \left(-500\left(\left(x-x_{e} / 2\right)^{2}+\left(y-y_{e} / 2\right)^{2}\right)\right)
$$

where $x_{e}=y_{e}=2$. The domain is consider to be $\left(0, x_{e}\right) \times\left(0, y_{e}\right)$ with Neumann boundary conditions. All other initial conditions are same as in the one-dimensional case. We consider two 

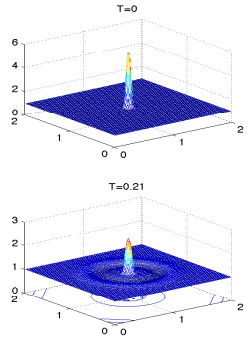
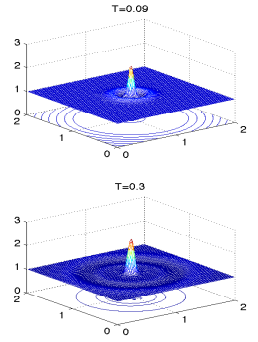

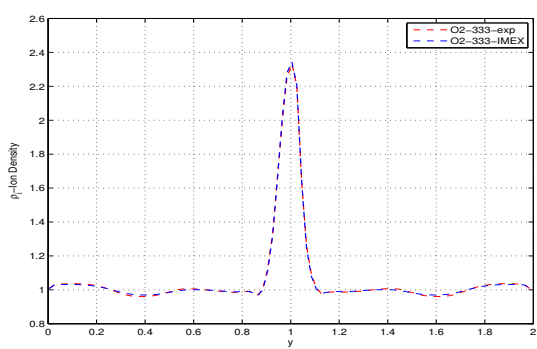

(a) Ion-density at non-dimensional time of $t=$ (b) Ion-density at non-dimensional time of $t=0.3$ 0, 0.09, 0.21 and 0.3 using O2-333-exp and HLLC using O2-333-exp and O2-333-imex schemes. Cut solver. 
333-imex is plotted in Figure 8(b) and 9(b) for $\hat{l}_{r}=10^{-2}$ and $\hat{l}_{r}=10^{-4}$ respectively. Similar to the $1 \mathrm{D}$ case we observe that IMEX scheme is slightly more diffusive then explicit scheme. The simulations time of the simulations are given in Table 2. We again note that IMEX scheme O2-333-imex is more efficient than explicit scheme O2-333-exp at $\hat{l}_{r}=10^{-4}$.

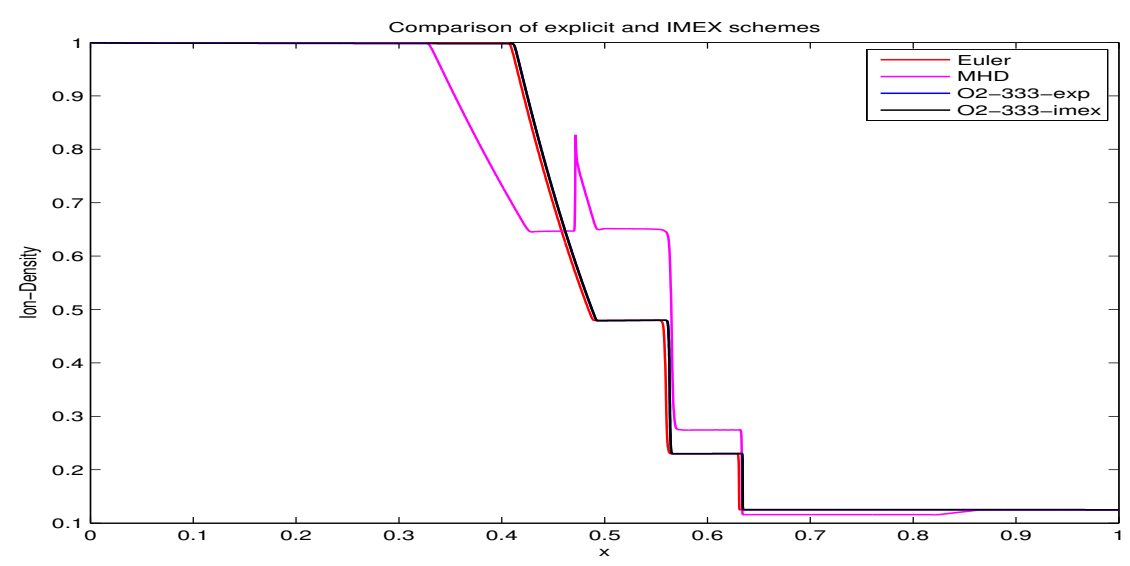

Figure 10. Generalized Brio-Wu Shock tube Problem: Comparison of Iondensity using O2-333-exp and O2-333-imex schemes. We use HLLC solvers with 10016 cells with $\hat{l}_{r}=100$. We have also plotted corresponding Euler fluid flow and ideal MHD solutions. Solution is close to the Euler fluid flow.

3.4. Generalized Brio-Wu shock tube Riemann problem. The Riemann problem is generalization of Brio-Wu shock tube Riemann problem for ideal MHD. We consider following left and right states as initial conditions:

\begin{tabular}{|c|c|c|c|c|c|c|}
\hline & $\rho_{i}$ & $p_{i}$ & $\rho_{e}$ & $p_{e}$ & $B^{x}$ & $B^{y}$ \\
\hline left & 1.0 & $5 \times 10^{-5}$ & $1.0 / m$ & $5 \times 10^{-5}$ & 0.75 & 1.0 \\
\hline right & 0.125 & $5 \times 10^{-6}$ & $0.125 / m$ & $5 \times 10^{-6}$ & 0.75 & -1.0 \\
\hline
\end{tabular}

on the computational domain $(0,1)$ with Neumann boundary conditions. All other variables are set to zero. The initial discontinuity is at $x=0.5$. We take realistic ion-electron mass ratio to be 1836. We non-dimensionalize the equations with reference pressure $p_{0}=10^{-4}$. Non-dimensional Debye length is taken to be 0.01 . We compute solutions by varying non-dimensional Larmor radius $\hat{l}_{r}$ between 100 and 0.001 . When $\hat{l}_{r}$ is high, source terms do not effect fluid flows, so we expect solution close to Euler fluid flow. As $\hat{l}_{r}$ is decreased, source terms starts to effects the flow and we starts to observe two fluid effects. As $\hat{l}_{r}$ tends to zero, we expect solution to come close to ideal MHD solution. This example will test the ability of the schemes to capture full spectrum of physics from pure fluid flow to two-fluid flow to ideal MHD. All the simulations are performed on 32 computational cores.

Results of simulations are presented in Figures 10, 11, 12, 13 and 14. For comparison we have also plotted corresponding Euler fluid flow and ideal MHD solutions. In Figure 10, we have plotted ion density for $\hat{l}_{r}=100$ on 10016 cells. In this case, source has no effect on the solution and flow behaves as pure fluid flow. Solutions for both O2-333-exp and O2-333-imex schemes are on the top of each other. Simulation time for O2-333-exp was 1421.11 seconds and for O2-333-imex was 1360.23 seconds. Hence both the schemes have similar performance.

In Figure 11, we have plotted solutions for $\hat{l}_{r}=1.0$ on 10016 cells. In this case, we are in two-fluid regime and source effects on the solutions are evident. In Figure 11(a), we have 


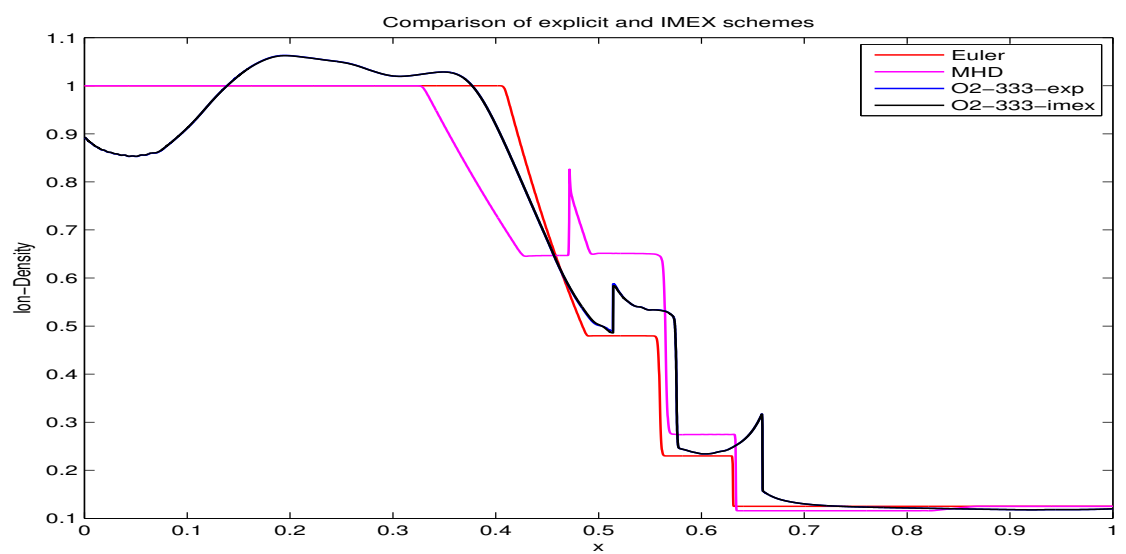

(a) Generalized Brio-Wu Shock tube Problem: Comparison of Ion-density using O2-333exp and O2-333-imex schemes at non dimensional time $t=0.1$.

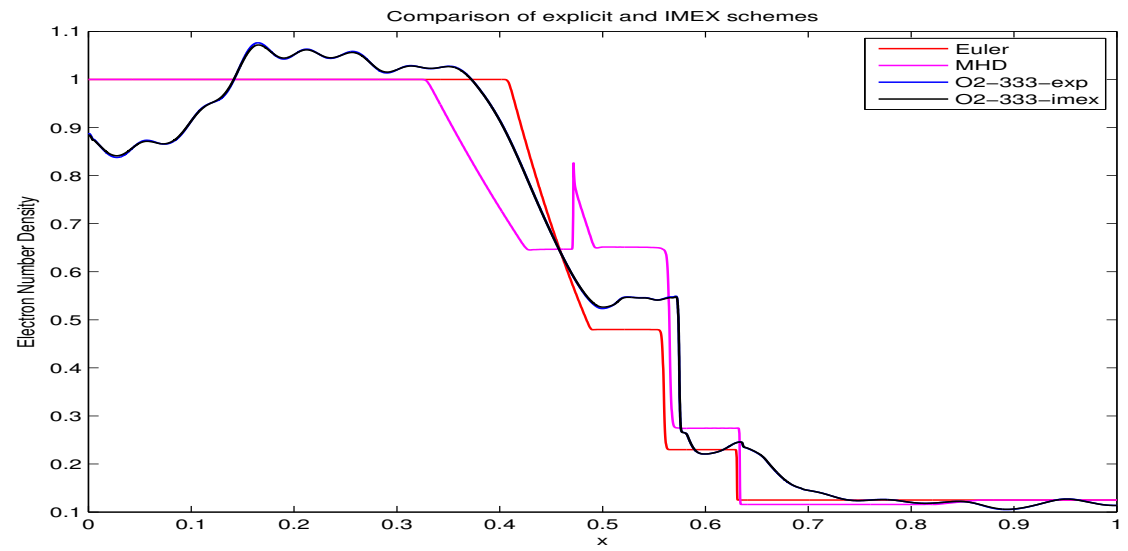

(b) Generalized Brio-Wu Shock tube Problem: Comparison of Electron number density using O2-333-exp and O2-333-imex schemes at non dimensional time $t=0.1$.

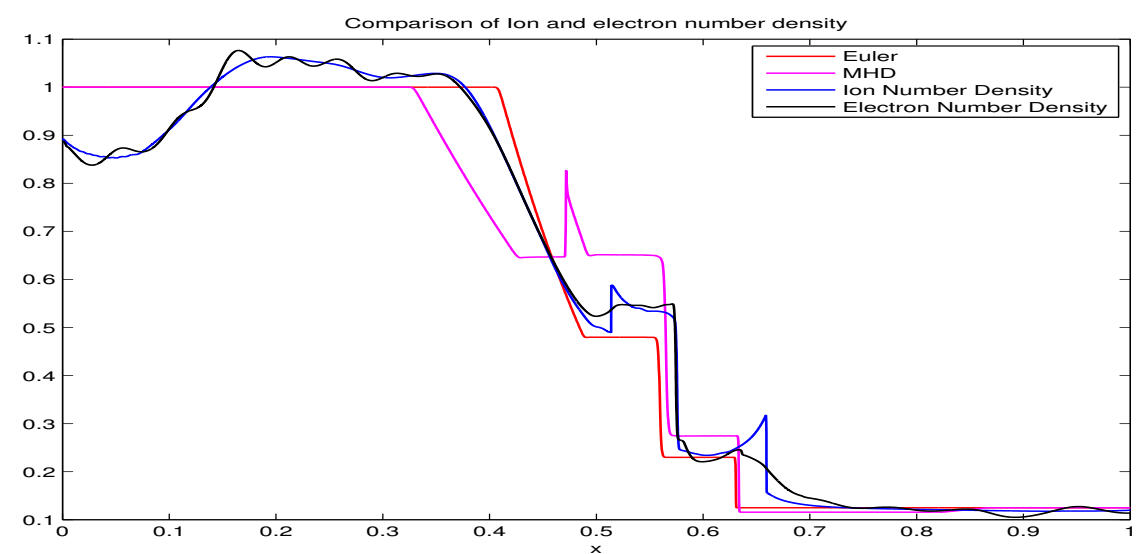

(c) Generalized Brio-Wu Shock tube Problem: Comparison of Ion and Electron number densities using O2-333-exp at non dimensional time $t=0.1$.

Figure 11. Generalized Brio-Wu Shock tube Problem: Comparison of O2333-exp and O2-333-imex schemes. We use HLLC solvers on 10016 cells with $\hat{l}_{r}=1.0$. We have also plotted corresponding Euler fluid flow and ideal MHD solutions. Solution is in Two-fluid regime and we can observe two-fluid effects. 


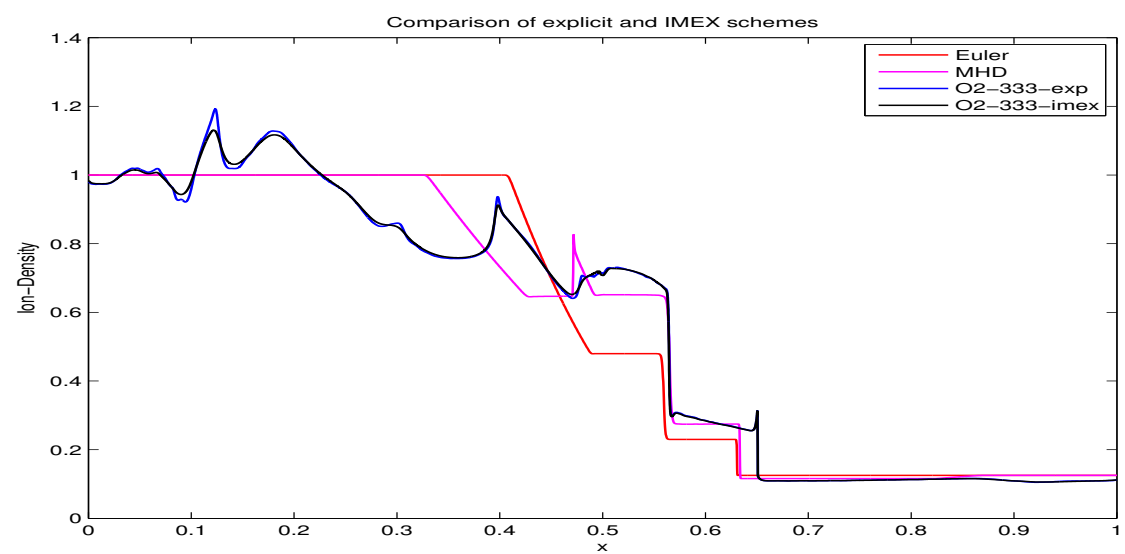

(a) Generalized Brio-Wu Shock tube Problem: Comparison of Ion-density using O2-333exp and O2-333-imex schemes at non dimensional time $t=0.1$.

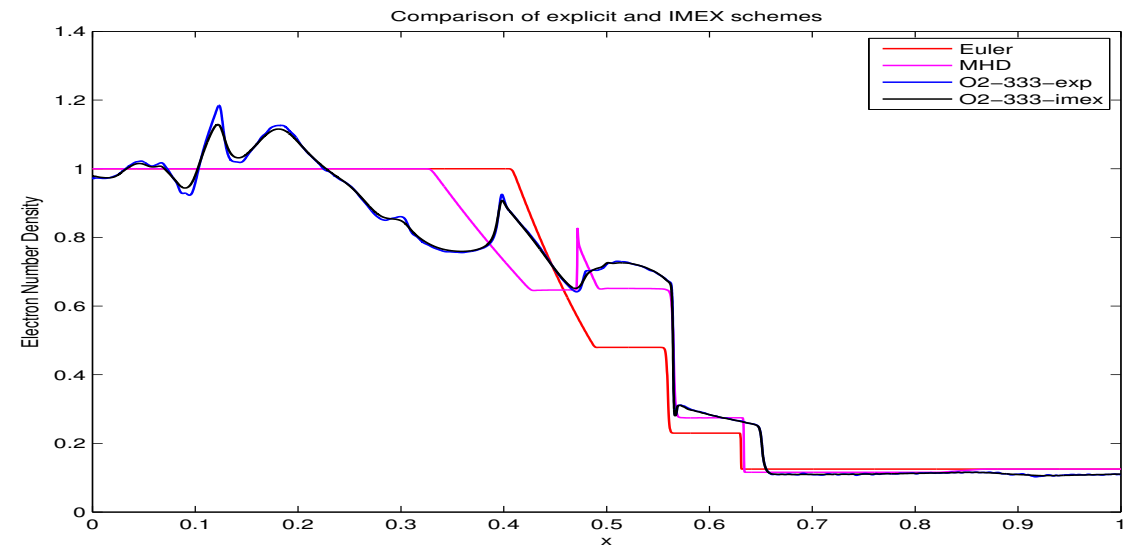

(b) Generalized Brio-Wu Shock tube Problem: Comparison of Electron number density using O2-333-exp and O2-333-imex schemes at non dimensional time $t=0.1$.

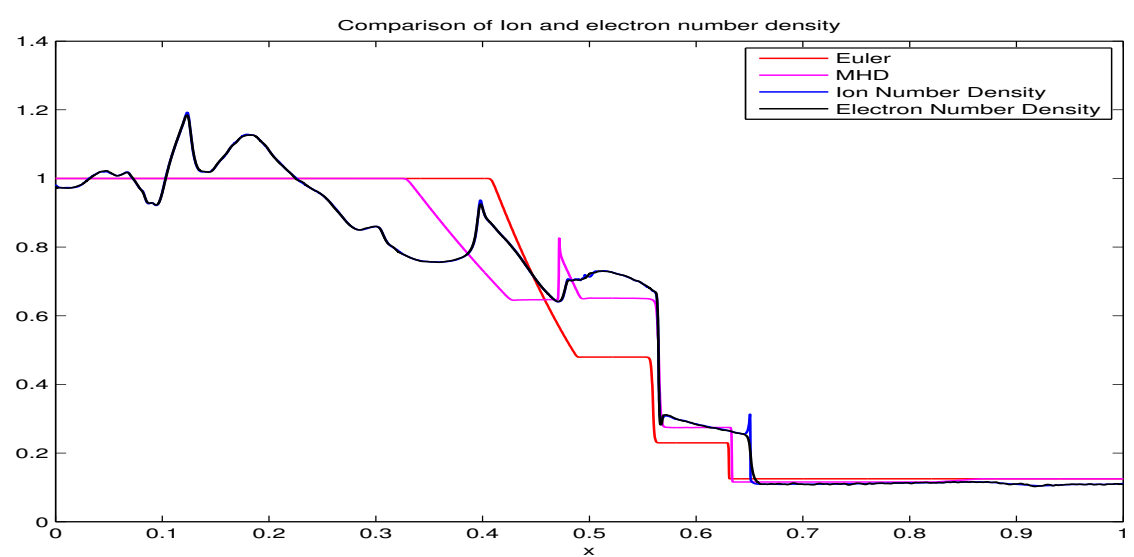

(c) Generalized Brio-Wu Shock tube Problem: Comparison of Electron and Ion number densities using O2-333-exp scheme at non dimensional time $t=0.1$.

Figure 12. Generalized Brio-Wu Shock tube Problem: Comparison of O2333-exp and O2-333-imex schemes. We use HLLC solvers on 10016 cells with $\hat{l}_{r}=0.1$. We have also plotted corresponding Euler fluid flow and ideal MHD solutions. Solution is in Two-fluid regime and we can observe two-fluid effects. 


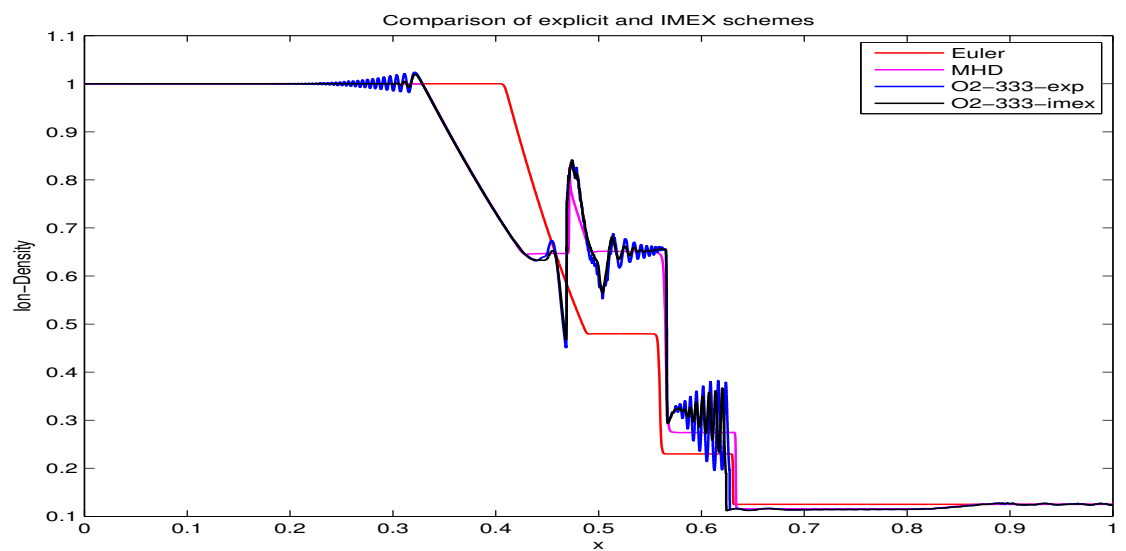

(a) Generalized Brio-Wu Shock tube Problem: Comparison of Ion-density using O2-333exp and O2-333-imex schemes at non dimensional time $t=0.1$.

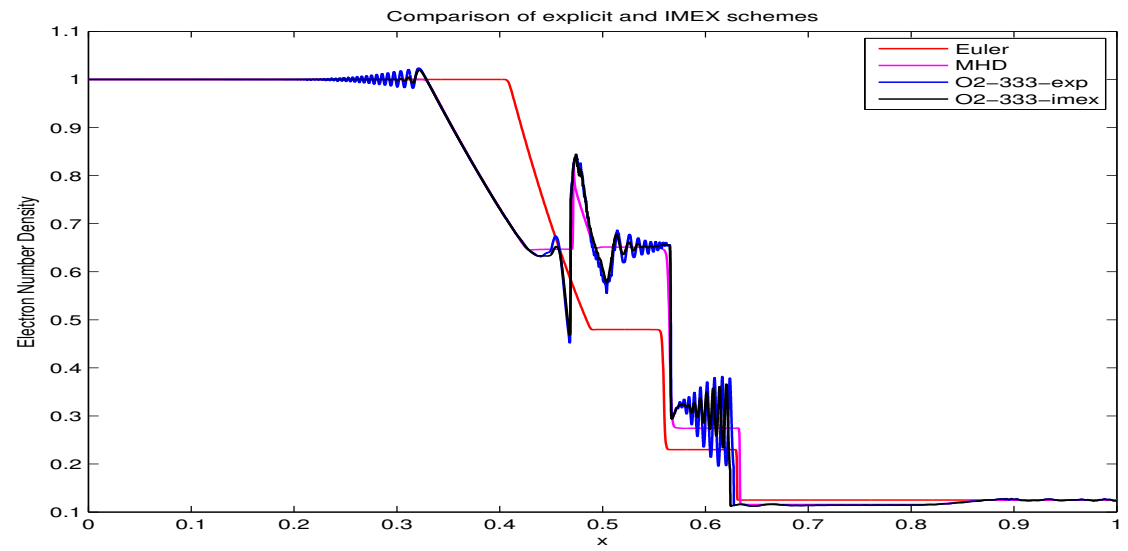

(b) Generalized Brio-Wu Shock tube Problem: Comparison of Electron number density using O2-333-exp and O2-333-imex schemes at non dimensional time $t=0.1$.

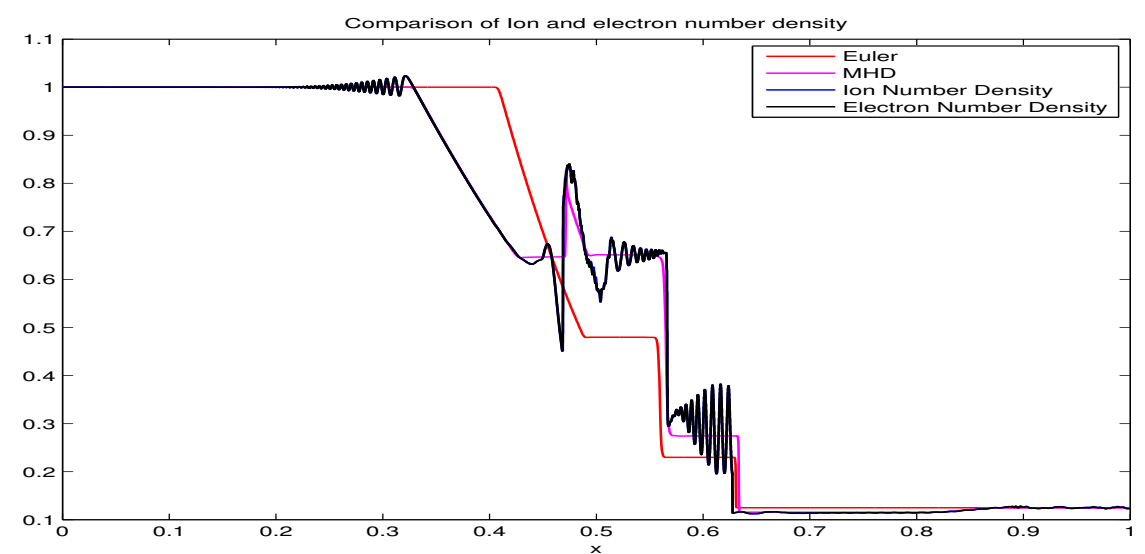

(c) Generalized Brio-Wu Shock tube Problem: Comparison of Electron and Ion number densities using O2-333-exp scheme at non dimensional time $t=0.1$.

Figure 13. Generalized Brio-Wu Shock tube Problem: Comparison of O2333-exp and O2-333-imex schemes. We use HLLC solvers on 50016 cells with $\hat{l}_{r}=0.001$. At this resolution all the MHD waves and additional two-fluid effects are resolved. 
compared the Ion-densities of the two schemes. We note that the O2-333-imex scheme is slightly more diffusive than O2-333-exp scheme, similar to results we have observe in the case of soliton simulations. In Figure 11(b), we observe the similar behavour of the two schemes, where we have compared electron number densities. Electron number density was computed by multiplying electron density with ion-electron mass ratio. To compare the ion and electron number densities, we have plotted them in Figure 11(c) using O2-333-exp scheme. Both ion and electron number densities are away from pure fluid flows and ideal MHD case. Furthermore, number densities are close to each other, but the profile differs as fluid is still not quasi-neutral. These results are similar to simulation in [9]. The performance of the explicit scheme O2-333-exp is comparable to the wave propagation schemes in [9]. The simulation time for O2-333-exp was 1240.5 second and for O2-333-imex was 1198.6 seconds.

Figure 12 has the solutions corresponding to the Larmor radius $\hat{l}_{r}=0.1$ on 10016 cells. Here, we have compared ion-densities and electron number densities for the O2-333-exp and O2-333imex schemes ( see Figure 12(a) and 12(b)). Again, we observe that IMEX scheme is slightly more diffusive than explicit scheme. In addition, from Figure 12(c) we note that number densities of ion and electron are much closer than the previous case and fluid appears to be quasi-neutral. Also number densities have moved closer to MHD solution. At this resolution simulation time for O2-333-exp was 1232.9 second and for O2-333-imex was 1197.8 seconds, which are comparable.

To simulate flow close to MHD regime, we further decrease the Larmor radius and consider $\hat{l}_{r}=0.001$. At this level we expect many small scale oscillations in the solution. To capture all the waves and oscillations we take 50016 cells (see [9, 12]). In Figure 13(a), we have compared ion densities from both schemes. Both O2-333-exp and O2-333-imex are able the resolve small scale oscillations and MHD waves, however explicit scheme is better at resolving the solutions. We observe similar situation in Figure 13(b). Number densities for O2-333-exp are compared with Euler and MHD simulation in Figure 13(c). We note solution has converged to the MHD case and only difference is small scale oscillation which are presented due to the very stiff source terms and they represent two-fluid physics at this scale. The simulations times for this case are 75022.7 seconds and 72853.1 seconds for O2-333-exp and O2-333-imex schemes, respectively.

Although, we are able to capture all the two-fluid effects using the highly resolved mesh, the computational costs was very high. Furthermore, it might not always be desirable to capture all the small scale oscillations. So, we need to have stable method where we can compute using coarser mesh and still get the reasonable results. However, at the lower resolutions when we use HLLC flux for each fluid component simulations becomes unstable. To overcome this difficulty we treat whole fluid part as one system and use HLLE solver for it. This implies replacing HLLE speeds for the ion fluid with that of electron fluid (which are much faster) and amount to adding further diffusion to the solver. We will represent this solver with mod-HLLE solver. Note that this solver still guarantees $\Omega$-invariance of the scheme as speeds still satisfies required conditions. This solver is very Robust at low resolutions.

In Figure 14, we have plotted the results using mod-HLLE solver on 1024 cells for $\hat{l}_{r}=0.001$. We note that both O2-333-exp and O2-333-imex are still able to capture MHD effects and additional two-fluid effects have been diffused. Again, explicit scheme is slightly better than IMEX scheme. At this resolution simulation time is dictated by the source terms. Hence IMEX schemes are more efficient. The computational time for O2-333-exp 50.13 seconds and for O2333-imex it is 21.85. Hence IMEX schemes are at least twice as fast as the explicit schemes.

\section{Conclusion}

We have designed $\Omega$-invariant schemes for the two-fluid plasma flow equations. For the explicit schemes we have obtained exact condition on time step for solution to be $\Omega$-invariant. As source terms can be stiff we have proposed IMEX schemes. For IMEX schemes we show that source 


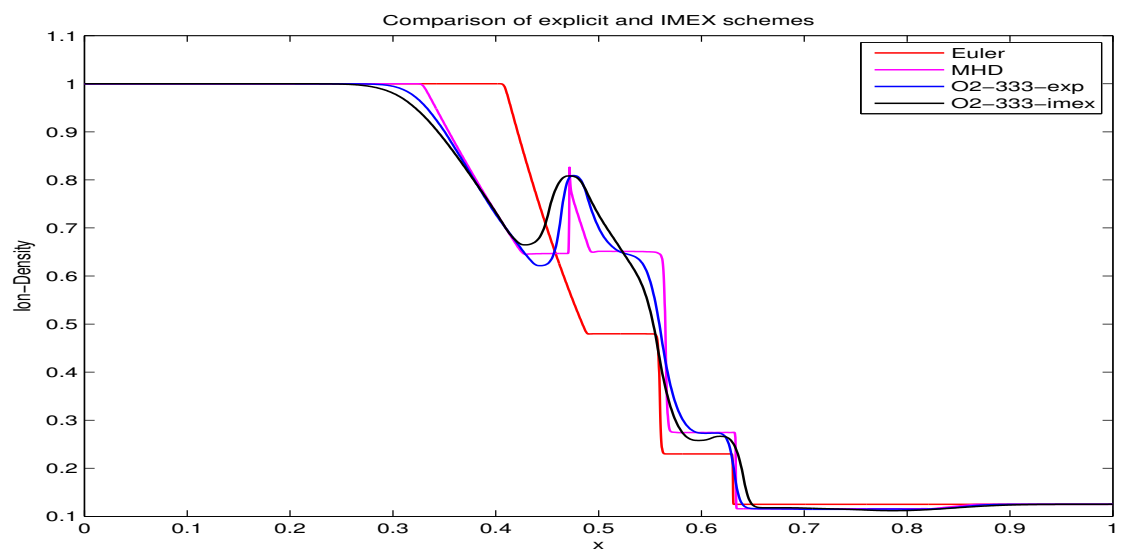

(a) Generalized Brio-Wu Shock tube Problem: Comparison of Ion-density using O2-333exp and O2-333-imex schemes at non dimensional time $t=0.1$.

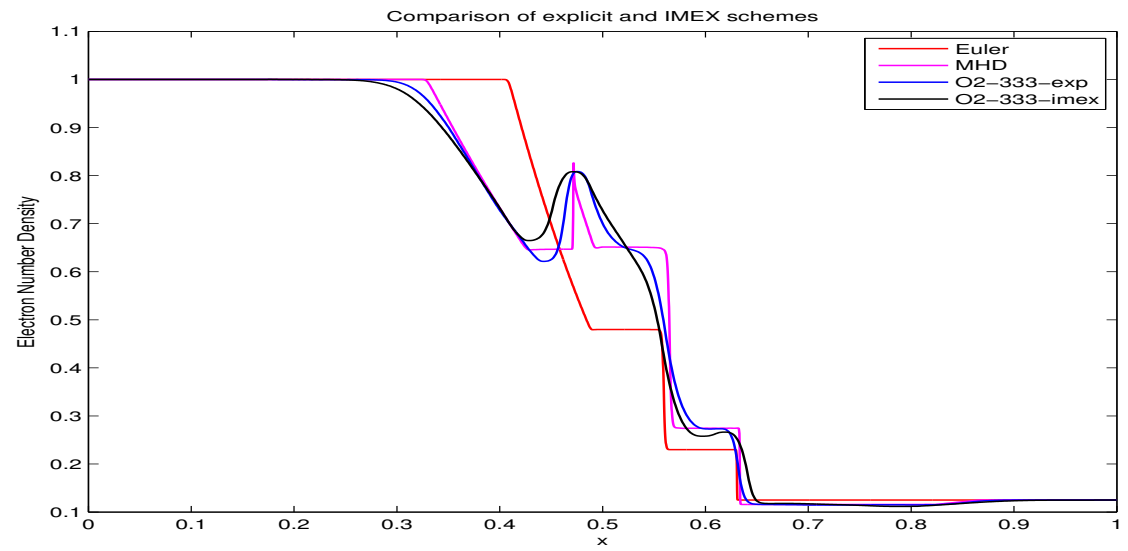

(b) Generalized Brio-Wu Shock tube Problem: Comparison of Electron number density using O2-333-exp and O2-333-imex schemes at non dimensional time $t=0.1$.

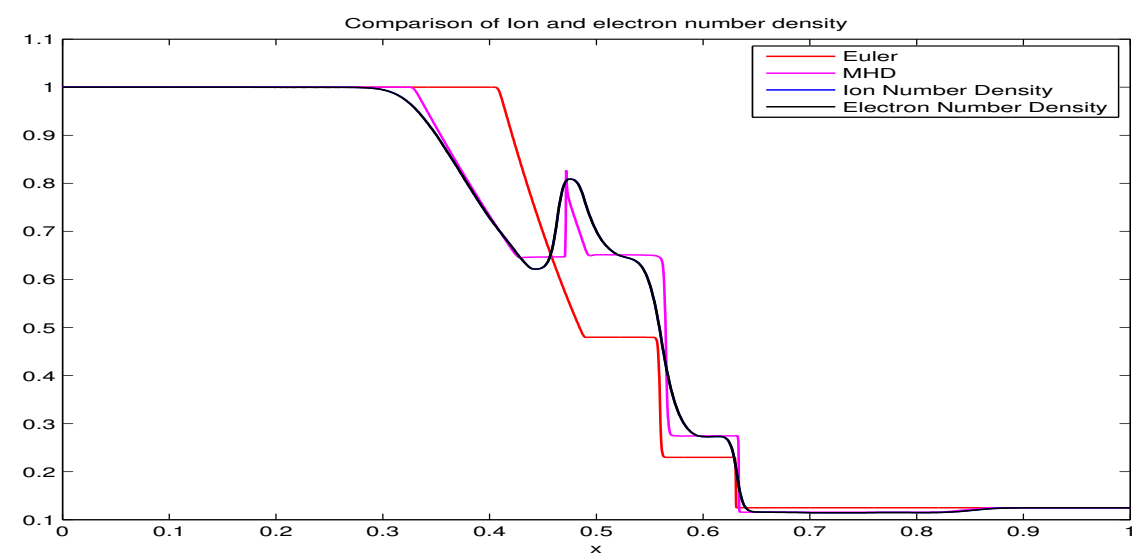

(c) Generalized Brio-Wu Shock tube Problem: Comparison of Electron and Ion number densities using O2-333-exp scheme at non dimensional time $t=0.1$.

FiguRe 14. Generalized Brio-Wu Shock tube Problem: Comparison of O2-333exp and O2-333-imex schemes. We use mod-HLLE solvers on 1024 cells with $\hat{l}_{r}=0.001$. At this resolution we ave resolved all the MHD waves and additional two-fluid effects are not seen. 
discretzation is unconditionally $\Omega$-invariant. These schemes are then applied to a set of numerical experiments to demonstrate robustness of these schemes. We observe that IMEX schemes are more diffusive than the explicit schemes. However for the low Larmor radius cases IMEX schemes are shown to highly efficient.

\section{ACKNOWLEDGEMENT}

R. Abgrall has been funded in part by EU ERC Advanced grant "ADDECCO" \#226616. H. Kumar has been funded by EU ERC Advanced grant "ADDECCO" \#226616 during this work.

\section{REFERENCES}

[1] Berthon, C., Stability of the MUSCL schemes for the Euler equations. Commun. Math. Sci., Vol. 3 (2), 133-157 (2005).

[2] Berthon, C., Why the MUSCL-hancock scheme is L1-stable. Numer. Math., Vol. 104 (1), 27-46 (2006).

[3] Bouchut, F., Nonlinear stability of finite volume methods for hyperbolic conservation laws and well-balanced schemes for sources. Frontiers in Mathematics, Birkhauser, Basel (2004).

[4] Baboolal, S., Finite-difference modeling of solitons induced by a density hump in a plasma multi-fluid. Math. and Comp. in Simulation, Vol. 55, 309-316 (2001).

[5] Baboolal, S., High-resolution numerical simulation of 2D nonlinear wave structures in electromagnetic fluids with absorbing boundary conditions. J. Comp. Appl. Math., Vol. 234, 1710-1716 (2010).

[6] Baboolal, S., Bharuthram, R., Two-scale numerical solution of the electromagnetic two-fluid plasma-Maxwell equations: Shock and soliton simulation. Math. and Comp. in Simulation, Vol. 76, 3-7 (2007).

[7] Gottlieb, S., Shu, C. W., Tadmor, E., Strong stability-preserving high-order time discretization methods. SIAM Rev., Vol. 43, 89-112 (2001).

[8] Goedbloed, H., Poedts, S., Principles of Magnetohydrodynamics. Cambridge University Press, Cambridge (2004).

[9] Hakim, A., Loverich, J., Shumlak, U., A high-resolution wave-propagation scheme for ideal two-fluid plasma equations. J. Comp. phys., Vol. 219, 418-442 (2006).

[10] Johnson, E.A., Rossmanith, J.A., Collisionless Magnetic Reconnection in a Five-Moment Two-Fluid ElectronPositron Plasma. Proceedings of Symposia in Applied Mathematics (proceedings of HYP2008), Volume 67.2, (2009).

[11] LeVeque, R. J., Finite Volume Methods For Hyperbolic Problems. Cambridge University Press, Cambridge (2002).

[12] Kumar, H. and Mishra, S., Entropy stable numerical schemes for two-fluid plasma equations. J. Sci. Comp., Vol. 52-2, 401-425 (2012).

[13] Loverich, J., Hakim, A. and Shumlak, U., A discontinuous Galerkin method for ideal two-fluid plasma equations. Arxiv preprint, arXiv:1003.4542, (2010).

[14] Munz, C. D., Omnes, P., Schneider R., Sonnendrüker, E. and Voß, U. Divergence correction techniques for Maxwell solvers based on a hyperbolic model. J. Comp. Phys., Vol. 161, 484-511 (2000).

[15] Shumlak, U. and Loverich, J., Approximate Riemann solvers for the two-fluid plasma model. J. Comp. Phys., Vol. 187, 620-638 (2003).

[16] Shu, C. W., TVD time discretizations. SIAM J. Math. Anal., Vol. 14, 1073-1084 (1988).

[17] Zhang, X. and Shu, C.W., On positivity-preserving high-order Discontinuous Galerkin schemes for Euler equations on rectangular meshes. J. Comp. Phys., Vol. 229, 8918-8934, (2010).

[18] Leer, B. Van, Towards the ultimate conservative difference scheme. V. A second-order sequel to Godunov's method, J. comput. Phys., Vol. 32, 101-136 (1979).

[19] Toro, E. F., Riemann solvers and numerical methods for fluid dynamics. A practical introduction Second edition. Springer-Verlag, Berlin (1999).

[20] Batten, P., Clarke, N., Lambert, C. and Causon, M., On the choice of wavespeeds for the HLLC Riemann solver. SIAM Journal on Scientific Computing, Vol. 18, 15-53 (1997).

[21] Srinivasan, B., Hakim, A., and Shumlak, U., Numerical Methods for Two-Fluid Dispersive Fast MHD Phenomena. Comm. in Comp. Phys., Vol. 10, 183-215 (2011). 
(Remi Abgrall)

BACCHUS TEAM,

INRIA, BORDEUAX,

FRANCE.

E-mail address: remi.abgrall@inria.fr

(Harish Kumar)

Department of Mathematics

IIT DELHI,

New-Delhi, India

E-mail address: hkumar@maths.iitd.ac.in 